\title{
Improvements of Film Cooling Effectiveness by using Barchan Dune Shaped Ramps
}

\author{
Wenwu Zhou and Hui Hu( $\bowtie)$ \\ Department of Aerospace Engineering, Iowa State University \\ 2271 Howe Hall, Room 1200, Ames, IA 50011, USA \\ Email: huhui@iastate.edu
}

\begin{abstract}
In the present study, an experimental investigation was conducted to explore a novel film cooling design with Barchan-dune-shaped-ramp (BDSR) concept to augment the effectiveness of film cooling injection from discrete holes. Inspired by the unique shape of Barchan dunes commonly seen in deserts to prevent sand particles on the ground from being blown away by the oncoming airflow, the BDSR concept was proposed to enable the coolant streams exhausted from coolant injection holes stay more firmly over the surface of interest for improved film cooling performance. During the experiments, while coolant streams were injected from discrete circular holes on a flat test plate at an injection angle of 35 degrees, a row of BDSRs were mounted on the test plate at either downstream or upstream of the coolant injection holes for improved film cooling effectiveness. While a high-resolution Particle Image Velocimetry (PIV) system was used to conduct detailed flow field measurements to quantify the dynamic mixing process between the coolant jet streams and the mainstream flows over the test plate, a Pressure Sensitive Paint (PSP) technique was used to map the corresponding adiabatic film cooling effectiveness on the surface of interest based on a mass-flux analog to traditional temperature-based cooling effectiveness measurements. The cooling effectiveness data of the BDSR design were compared quantitatively against those of a conventional film cooling design without BDSR (i.e., baseline case) under the same test conditions in order to evaluate the effects of the BDSRs on the film cooling effectiveness over the surface of interest. The effects of the locations and height of the BDSRs in relation to the coolant injection holes on the film cooling effectiveness at different blowing ratios (i.e., the coolant-to-mainstream mass flux ratios) were examined quantitatively based on the PIV and PSP measurements. The detailed flow field measurements were correlated with the measured film cooling effectiveness distributions to elucidate the underlying physics in order to explore/optimize design paradigms for better film cooling protection of turbine blades from harsh environments.
\end{abstract}

\section{INTRODUCTION}

Because of wide-spreading application of gas turbine engines in power and transportation industries, there is a great incentive to maximize the performance of turbine engines for both economic and environmental reasons. Efficiency improvements of gas turbine engines, even if only by marginal increments, will lead to significant value of savings. Thermodynamic analysis reveals that the thermal efficiency and power output of a gas turbine can be greatly enhanced through a higher turbine inlet temperature. Therefore, means to protect the hot components of gas turbines from corrosion and melting are very essential, especially the implementation of film cooling technique. While coolant bleeds represent energy loss which should be minimized, gas turbine designers are continuously seeking to improve film cooling efficiency without increasing the consumption of coolant flow.

The film cooling effectiveness is found to be affected by a series of parameters [1,2], including mass flux ratio (i.e., blowing ratio, $M$ ), momentum flux ratio (I), coolant to mainstream density ratio (DR), turbulence intensity levels of the oncoming mainstream flows, and geometric shapes of the coolant 
injection holes. Film cooling, fundamentally, is a jet-in-cross-flow (JICF) problem. As described in Fric \& Roshko [3], the dominated flow features of a JICF include shear-layer-related vortices formed at the leading edge of the jet, horseshoe vortices around the jet, counter-rotating-vortex (CRV) pair in the jet, and wake vortices downstream of the jet. The performance of a film cooling design is closely related to how firmly the coolant stream can stay attached to the surface of interest after exhausted from the coolant injection hole. A major problem of film cooling is that the coolant stream tends to detach from the surface of interest due to the vortex induction from the CRV structures in the jet flow, which leads to deteriorated film cooling performance. Extensive experimental and numerical studies have been conducted in recent years to explore new film cooling designs for improved film cooling effectiveness. For example, Goldstein et al. [4] reported a great enhancement of film cooling effectiveness by using fan-shaped holes. Their flow visualization results revealed that the coolant jet exhausted from a fan-shaped hole would stay close to the wall surface, unlike the coolant stream injected from a conventional circular hole which would detach from the surface of interest at high blowing ratios. Zaman et al. [5] experimentally studied an inclined jet flow interacting with a vortex generator, and showed that the vortex generator would produce a vorticity cancellation effect that can postpone the jet lifting off from the wall surface, thereby, better film cooling performance. By conducting a large eddy simulation (LES) study on a film cooling flow with a micro-ramp vortex generator, Shinn et al. [6] found that the strength of counter-rotating vortex (CRV) structures in the coolant jet flows would be reduced substantially due to the exist of the vortex generator, resulting in a better film cooling effectiveness over the surface of interest. More recently, An et al. [7] conducted an experimental study to investigate the film cooling performance behind a crescent block, and showed that the film cooling effectiveness would become better within certain range of blowing ratios due to the existence of the block. Na \& Shih [8] performed a numerical study on a new film cooling configuration with a ramp placed upstream of a coolant injection hole, and found that the backward-facing ramp can greatly enhance the film cooling effectiveness over the surface of interest. Sakai et al. [9] investigated the combined effects of internal ribs and downstream bumps (semicircular, hemispherical and cylindrical bumps) on film cooling effectiveness. Their measurements showed that the film cooling effectiveness of the studied configurations would be strongly affected by both the rib orientations and bump shapes. While most of the ramp/bump designs suggested in those previous studies are shown to be effective in improving film cooling performance, such ramp/bump designs with sharp edges could cause high skin friction, thereby, increased aerodynamic losses. More comprehensive reviews about different film cooling designs can be found in the articles of Goldstein[10] and Bunker[11].

Barchan dunes, which are commonly seen in deserts, are featured by a crescentic platform and a streamlined shape along the wind flow direction in both of the windward side (convex face) and leeward side (concave face) along with two gradually-widened horns pointing downstream [12,13]. When an airflow passes over a Barchan dune, a pair of symmetric vortices are found to form in the wake of the dune [14,15]. Induced by the arched shape of the dune, the formed vortex pair can generate a continual suction along the axis of the horns, creating a "quiet" wake flow behind the dune. As a result, sand particles can settle down on the ground behind the dune, instead of being blown away from the ground by the oncoming airflow. It should also be noted that the streamlined shape of Barchan dunes has also been found to be able to minimize the friction losses of the airflow as passing over the dunes. Inspired by the unique feature of Barchan dunes to prevent sand particles on the ground from being blown away by the oncoming boundary layer airflow, a new film cooling design with Barchan-dune-shaped-ramp (BDSR) concept is proposed here to enable the coolant streams exhausted from coolant injection holes stay more firmly over the surface of interest for improved film cooling performance.

In the present study, an experimental investigation was conducted to explore a novel film cooling design with Barchan-dune-shaped-ramp (BDSR) concept for improved film cooling performance by taking advantage of the unique flow structures induced by Barchan dunes. The experimental study was conducted in a low-speed, open-circuit wind tunnel available at Iowa State University. While coolant streams was injected from discrete circular holes on a flat plate at an injection angle of 35 degrees, a row 
of BDSRs were mounted on the test plate at either downstream or upstream of the coolant injection holes. During the experiments, a high-resolution stereoscopic Particle Image Velocimetry (PIV) system was used to conduct detailed flow field measurements to quantify the dynamic mixing process between the coolant jet streams and the mainstream flows for the test cases with BDSRs mounted onto the test plate, in comparison to a baseline case (i.e. without the BDSRs mounted on the test plate). A Pressure Sensitive Paint (PSP) technique was used to map the corresponding adiabatic film cooling effectiveness on the surface of interest, based on a mass-flux analog to traditional temperature-based cooling effectiveness measurements. The effects of the location and height of BDSRs on the film cooling effectiveness over the surface of interest at different blowing ratios were examined quantitatively based on the PIV and PSP measurements. The detailed flow field measurements were correlated with the measured film cooling effectiveness distributions to elucidate the underlying physics in order to explore/optimize design paradigms for better film cooling protection of turbine blades from harsh environments

It should be noted that recent advances in additive manufacture (i.e., 3D-printing) technology have greatly broadened the horizon of film cooling designs. Many innovative-but-traditionally-unmachinable film cooling designs (e.g., the innovative film cooling design with BDSR to be explored in the present study) can now be manufactured easily as an integrated part at engine scale, which greatly promotes the development of film cooling technology. There are also several advantages for the proposed BDSR concept, in comparison with conventional film cooling designs. For example, the BDSR design can provide an alternative choice to increase the injection angle of cooling streams and make coolant streams to stay attached onto the surfaces of interest more firmly. It would also offer a flexible option to place BDSRs at either upstream or downstream of coolant injection holes. While most of turbine blades of modern gas turbines are coated with thermal barrier coatings (TBCs), it is possible to utilize TBCs to make tiny BDSRs on the surfaces of blades through an additive manufacture method to improve film cooling effectiveness for an even better protection to the turbine blades from overheating.

\section{EXPERIMENTAL SETUP AND TEST MODELS}

\subsection{Experimental models and test rig.}

The experimental study was conducted in a low-speed, open-circuit wind tunnel located at the Department of Aerospace Engineering of Iowa State University. The wind tunnel has an opticallytransparent test section with a cross section of $200 \mathrm{~mm} \times 125 \mathrm{~mm}$ and is driven by an upstream blower. With honeycombs and screen structures installed ahead of contraction section, the wind tunnel can supply uniform low-turbulence oncoming flow into the test section. The turbulence intensity of mainstream airflow in the test section was found to be about $1.5 \%$, as measured by using a hotwire anemometer.

In the present study, all test models including test plate and BDSRs were made of a hard plastic material and manufactured by using a rapid prototyping machine (i.e., 3-D printing) that built the models layer-by-layer with a resolution of about 25 microns. Figure 1 shows the film cooling design with BDSR concept. A row of 5 discrete circular holes with a same diameter of $D=4.0 \mathrm{~mm}$ were designed to inject coolant streams onto a flat plate at an injection angle of 35 degrees. While the entry length of the coolant injection holes is $4 D$, the spanwise pitch between the adjacent holes was designed to be $3 D$. The axial centerlines of the coolant injection holes intersect the upper surface of the test model at a distance of $L_{\text {leading }}=112.0 \mathrm{~mm}$ from the leading edge of the test plate. Thus, the boundary layer develops for a length of 22D before the mainstream flow encounters with the coolant injection holes. During the experiments, the test plate was flush-mounted to the bottom wall of the wind tunnel test section.

A stripe of 1.0 inch wide and 32 grit sand paper was employed at the leading edge of test section to trip the oncoming boundary layer to ensure a fully developed turbulent boundary layer flow over the flat plate. During the experiments, the mainstream flow velocity at the inlet of the test section was fixed at $U_{\infty}=25 \mathrm{~m} / \mathrm{s}$, and the corresponding Reynolds number is $R e=1.8 \times 10^{5}$, based on the distance between the 
leading edge of the test plate and the coolant injection holes. Figure 2 shows the measured boundary layer profiles at the immediate upstream of the coolant injection holes for the baseline case (i.e., without BDSRs mounted onto the test plate). Based on the measurement results as those shown in Fig. 2, the thickness of the turbulent boundary layer was estimated, which was found to be $\delta_{99} \approx 1.4 D$, and corresponding momentum thickness $\theta \approx 0.15 D$ (corresponding Reynolds number of $R e_{\theta} \approx 960$ ), and shape factor $H \approx 1.32$.

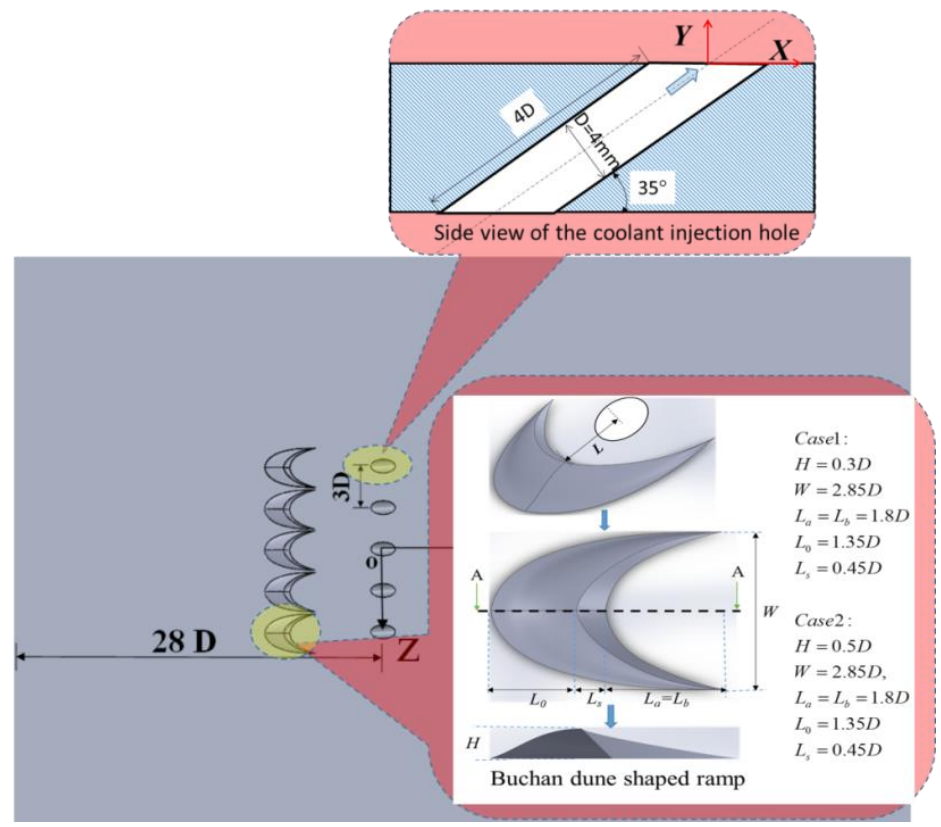

Figure 1. The schematic of BDSR assisted film cooling design ( $D$ is the diameter of coolant hole).

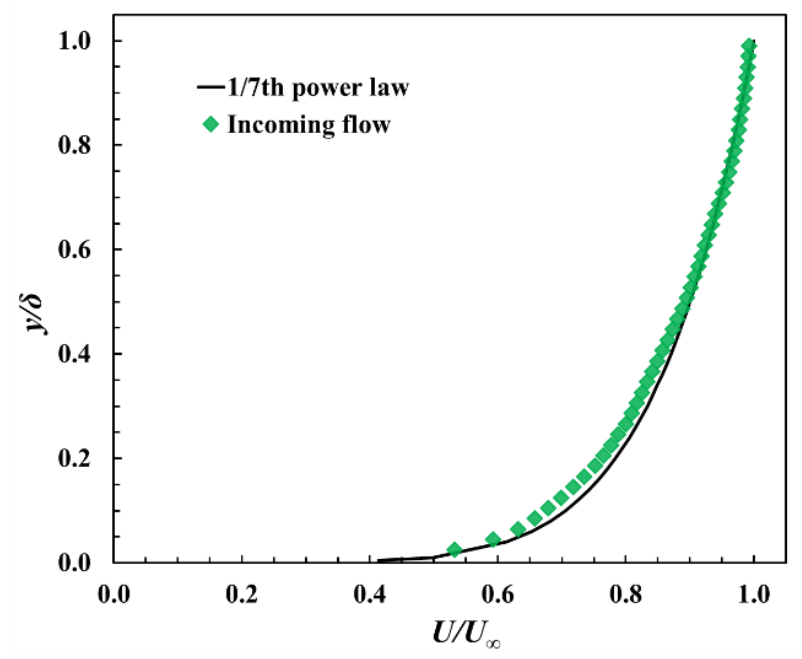

Figure 2. Measured turbulent boundary layer profile of the mainstream flow over the test plate.

It should be noted that, the size and shape of the Barchan dunes existing in nature are found to vary in a wide range, due to the environmental diversity (i.e., wind strength, moisture, sand supply and so on). The geometry of the BDSR models used in the present study was generated from a stereoscopic topographic map of an idealized Barchan dune reported in the previous studies [12,14,16,17]. As shown 
schematically in Fig. 1, five BDSRs were affixed onto the upper surface of the test plate at either downstream or upstream locations of the coolant injection holes by using a layer of $80 \mu \mathrm{m}$-thick double sided tape. During the experiments, the distance between the trailing edge of slip face and center of coolant hole, $L$, were adjusted for different test cases. The effects of the height of the BDSRs on the film cooling effectiveness over the test plate was also investigated by using two types of BDSRs, which shared similar geometry, but different height (i.e., $H=0.3 D$ and $0.5 D$, respectively).

\subsection{Adiabatic film cooling effectiveness measurement by using PSP technique.}

Adiabatic film cooling effectiveness $\eta$ is traditionally expressed as,

$$
\eta=\frac{T_{\infty}-T_{a w}}{T_{\infty}-T_{c}}
$$

where $T_{\infty}$ is the temperature of the main-stream, $T_{a w}$ is the adiabatic wall temperature of the surface under inspection, and $T_{c}$ is the hole exit temperature of the coolant stream.

In the present study, instead of conducting temperature measurements on the surface of interest (such as using thermocouples [18], liquid crystal thermometry [19], infrared thermography [20] or Temperature Sensitive Paint (TSP) [21]), the film cooling effectiveness measurements were performed at an isothermal condition by using a Pressure Sensitive Painting (PSP) technique [22] based on mass transfer analogy. As described in Johnson et al. [23], PSP based cooling effectiveness measurements can easily eliminate the concerns and implications associated with the effects of heat conduction through the test models on the adiabatic film cooling effectiveness measurements. With the value of the Lewis number $\left(\right.$ Le $\left.=\alpha / D_{s}\right)$ being about 1.0, the thicknesses of the thermal boundary layer and concentration boundary layer are of the same order, the differential equations regarding heat and mass transfer can be treated as analogous [21,24,25], which is the case of the present study ( $L e \approx 1)$.

For PSP experiments, the interested surface is coated with a layer of oxygen-sensitive paint. This paint consists of luminophores molecules hold together through gas-permeable polymeric binder. The luminophores molecules in PSP paint emit light when excited by certain UV light. However, the excited molecules will return to ground state via a reduced or radiationless emission in the presence of diatomic oxygen molecules. Such process is called oxygen quenching [22] where the intensity of the photoluminescence is inversely proportional to the concentration of the local oxygen. Consequently, the concentration of oxygen over the interested surface can be calculated based on the recorded light intensity through a calibration procedure. To replace the temperature terms with the concentrations of oxygen in Eq. (1), the adiabatic film cooling effectiveness can be expressed as:

$$
\eta=\frac{\left(C_{o_{2}}\right)_{\text {main }}-\left(C_{o_{2}}\right)_{\text {mix }}}{\left(C_{o_{2}}\right)_{\text {main }}-\left(C_{o_{2}}\right)_{\text {coolant }}}=\frac{\left(C_{o_{2}}\right)_{\text {main }}-\left(C_{o_{2}}\right)_{\text {mix }}}{\left(C_{o_{2}}\right)_{\text {main }}}
$$

For the choice of a coolant gas whose molecular mass differs greatly from that of the mainstream, the film cooling effectiveness based on the measurements of the concentration of oxygen should be expressed as Eq. (3), as suggested by Charbonnier et al [26], where $M W$ is the molecular weight ratio of the coolant gas to the mainstream gas.

$$
\eta=1-\frac{1}{\left[\left(\left(p_{o_{2}}\right)_{\text {air }} /\left(p_{o_{2}}\right)_{\text {mix }}\right)_{\text {wall }}-1\right] M W+1}
$$

The pressures terms in Eq. (3) can be determined by using the recorded intensity of emission light, which is directly related to the partial pressure of oxygen. The mathematic function between the normalized intensity and partial pressure can be obtained through a dedicated PSP calibration procedure. 
Further technical details about technical basis of PSP technique and PSP calibration procedure can be found in published literatures of Wright et al.[21], Yang and Hu[27], and Johnson et al.[23].

Figure 3 shows the schematic of the experimental setup used in the present study to map film cooling effectiveness distribution over the test plate by using the PSP technique. As shown in the figure, a UV LED light (LM2X-DM, ISSI) with wavelength of $390 \mathrm{~nm}$ was used as the excitation source for the PSP measurements. A 14-bit (2048 pixel $\times 2048$ pixel) charge-coupled device $(\mathrm{CCD})$ camera $(\mathrm{PCO} 2000$, Cooke Corp.) fitted with a $610 \mathrm{~nm}$ long-pass filter was used to records the intensity of the photoluminescence light emitted by excited PSP molecules. The PSP paint used in the study was Uni-FIB provided by ISSI. This type of PSP paint has a low sensitivity to temperature variation $\left(\sim 0.5 \% /{ }^{\circ} \mathrm{C}\right)$, making it an ideal candidate for the film cooling study. The experimental study was conducted in a room $\left(\sim 22^{\circ} \mathrm{C}\right)$ where the temperature fluctuation was found to be less than $\sim 0.5^{\circ} \mathrm{C}$.

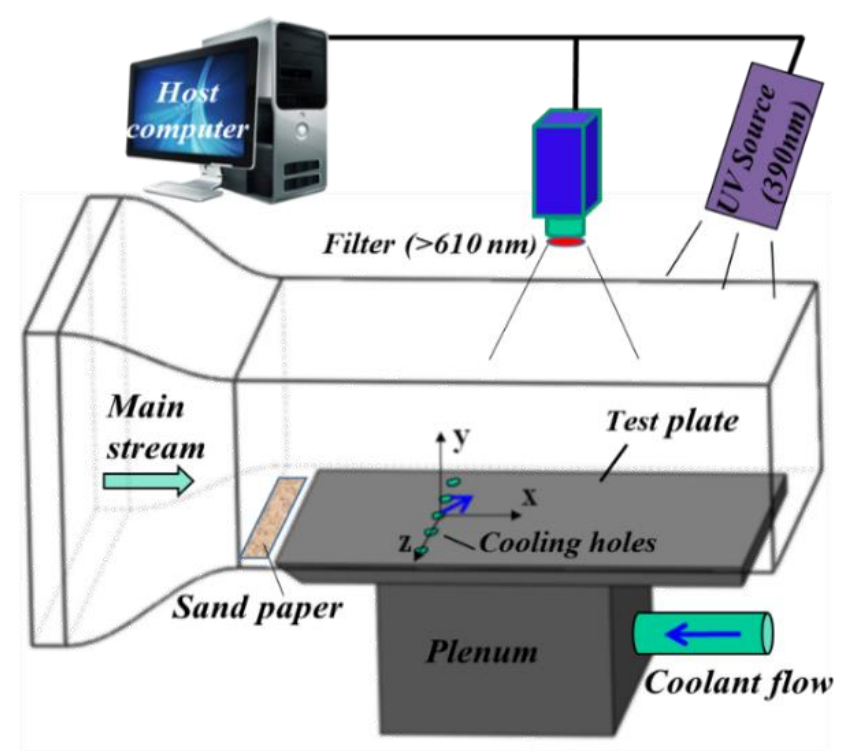

Figure 3. Experimental setup used for film cooling effectiveness measurements with PSP technique

During the experiments, while the airflow from the wind tunnel was used to simulate the hot gas flow in a typical turbine stage, an oxygen-free gas (i.e., $\mathrm{CO}_{2}$ with a density ration of $D R=1.5$ for the present study) was supplied to simulate the "coolant" stream for the PSP measurements. The oxygen-free coolant gas, which was supplied by a pressurized $\mathrm{CO}_{2}$ gas cylinder ( $99.99 \%$ purity), passed through a long pipeline and a constant temperature thermal bath before finally entering into the plenum chamber underneath the test plate. The blowing ratio (i.e., mass flux ratios, $M=\rho_{c} V_{c} / \rho_{\infty} V_{\infty}$ ) were monitored by using a flow meters (Omega, FMA-1600A), which were varied from 0.40 to 1.25. As a results, the corresponding momentum ratios $\left(I=\rho_{c} V_{c}^{2} / \rho_{\infty} V_{\infty}^{2}=M^{2} / D R\right)$ were ranging from 0.10 to 1.02 , and the velocity ratio $\left(V_{r}=V_{c} / V_{\infty}\right)$ changed from 0.26 to 0.82 .

In the present study, as suggested by Johnson \& Hu [28], a spatially-averaging procedure with interrogation windows of $9 \times 9$ pixels and 50\% overlap rate was performed for the acquired PSP images, in order to minimize the measurement uncertainties,. Since the acquired PSP images had a magnification of $0.07 \mathrm{~mm} /$ pix, the spatial resolution of the PSP measurement results would be about $0.30 \mathrm{~mm}$ (i.e., $0.07 D$ ). It is well known that the uncertainty of measured film cooling effectiveness by using PSP technique is closely related to the PSP calibration process, quality of the acquired raw images, and the flow conditions of the mainstream and coolant streams. Following up the work of Johnson \& Hu [28], the absolute measurement uncertainty of the present study was estimated to be within $\Delta \eta=0.02$ for the 
measured centerline film cooling effectiveness. The relative measurement uncertainty is estimated to be within $3 \%$ for $\eta=0.6$ and $5 \%$ for $\eta=0.4$. As for the laterally-averaged film cooling effectiveness, the absolute uncertainty would be within $\Delta \eta=0.03$, and the corresponding relative uncertainty is within $5 \%$ for $\eta=0.6$ and $7 \%$ for $\eta=0.4$. These uncertainty values were estimated based on a Taylor Series Method [29,30]. Further details about the uncertainty analysis of the measured film cooling effectiveness by using PSP technique can be found in Johnson \& Hu [28].

\subsection{Flow field measurements using the 2-D PIV and Stereoscopic PIV (SPIV) technique.}

In addition to the PSP measurements, a high-resolution Particle Image Velocimetry (PIV) system was also used in the present study to conduct detailed flow field measurements to quantify the dynamic mixing process between the coolant jet stream and mainstream flows over the test plate. Figure 4 shows the schematic of the experimental setup used for the PIV measurement. During the experiments, the mainstream airflow and the coolant gas stream (i.e., $\mathrm{CO}_{2}$ for the present study) were seeded with $\sim 1 \mu \mathrm{m}$ oil droplets generated by using droplet generators. Illumination was provided by a double-pulsed Nd:YAG laser (NewWave Gemini 200) emitting two pulses of $200 \mathrm{~mJ}$ at the wavelength of $532 \mathrm{~nm}$ with a repetition rate of $10 \mathrm{~Hz}$. By using a set of mirrors and optical lenses, the laser beam was shaped into a thin light sheet with a thickness of about $1.0 \mathrm{~mm}$ in the measurement region. The illuminating laser sheet was aligned along the flow direction of mainstream, bisecting the coolant hole in the middle of the test plate, to conduct 2D PIV measurements in the streamwise plane (i.e., within $X-Y$ plane) at first. Then, the laser sheet was rotated 90 degrees to perform stereoscopic PIV (SPIV) measurements at different downstream locations behind the exits of the coolant injection holes to reveal the flow structures in the cross plane normal to the mainstream flow direction (i.e., in the $Y-Z$ planes).

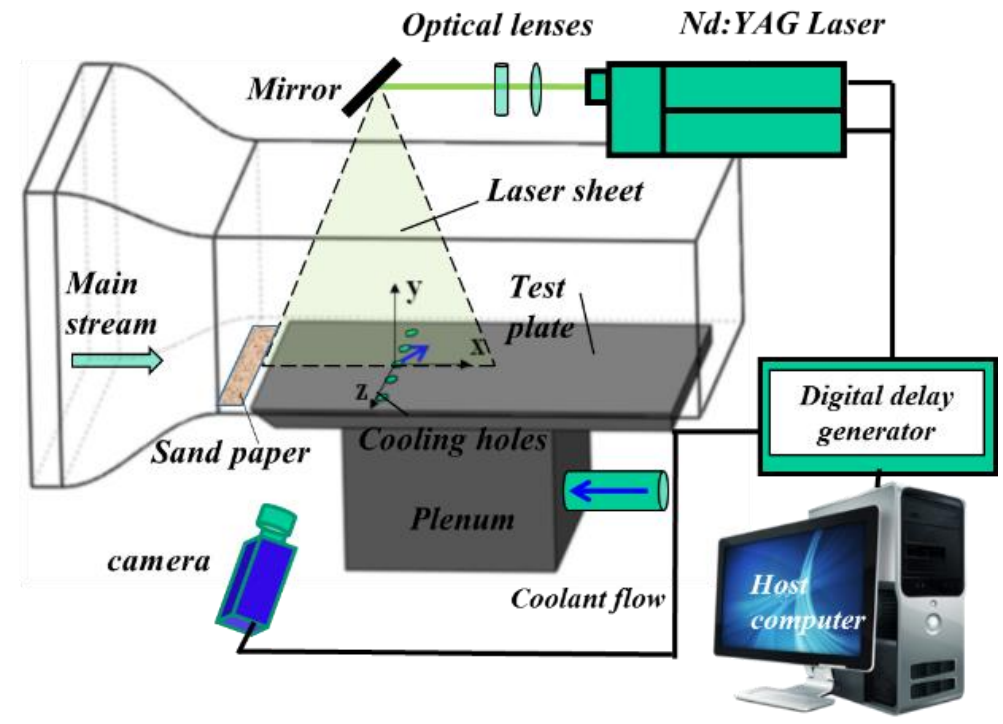

Figure 4. Experimental test rig for PIV measurements.

In the present study, two high resolution 14-bit high-resolution digital cameras (PCO2000, Cooke Corp.) were used for the SPIV image acquisitions. The two cameras were arranged with an angular displacement configuration of about $45^{\circ}$ in order to get a large overlapped view. With the installation of tilt-axis mounts, laser illumination plane, the lenses and the cameras were adjusted to satisfy the Scheimpflug condition. The cameras and double-pulsed Nd:YAG laser were both connected to a Digital Delay Generator (Berkeley Nucleonics, Model 565) to control the timing of the laser pulses and image 
acquisitions. A general in-situ calibration procedure was performed to obtain the mapping functions between the image planes and the object planes for the SPIV measurements. A target plate $(\sim 150 \mathrm{~mm} \times$ $150 \mathrm{~mm}$ ) with $0.5 \mathrm{~mm}$-diameter dots spaced at intervals of $1.0 \mathrm{~mm}$ was used for the in-situ calibration. The mapping function used in the present study was a multi-dimensional polynomial function, which is fourth order for the directions parallel to the laser illumination plane (i.e., $Y$-axis and $Z$-axis directions), and second order for the direction normal to the laser sheet plane (i.e., $X$-axis direction). The coefficients of the multi-dimensional polynomial were determined from the calibration images by using a least-square method as used by Hu et al. [31]

For the 2D PIV measurements in the streamwise plane, instantaneous flow velocity vectors were obtained by using a frame-to-frame cross-correlation technique to process the acquired PIV images with an interrogation window size of 32 pixels $\times 32$ pixels. An effective overlap of $50 \%$ of the interrogation windows was employed in PIV image processing, which results in a spatial resolution of $0.4 \mathrm{~mm}$ (i.e., $0.1 D$ ) for the PIV measurement results. Similar process methodology was also used for SPIV image processing. The instantaneous $2 \mathrm{D}$ velocity vectors were then used to reconstruct all three components of the flow velocity vectors in the laser illuminating plane by using the mapping functions obtained through the calibration procedure. After the instantaneous flow velocity vectors $\left(u_{i}, v_{i}, w_{i}\right)$ were determined, the distributions of the ensemble-averaged flow quantities such as mean velocity $(\mathrm{U}, \mathrm{V}, \mathrm{W})$, spanwise vorticity $\left(\omega_{z}\right)$ for the 2D PIV measurements in the streamwise plane and streamwise vorticity $\left(\omega_{x}\right)$ for the SPIV measurements in the cross planes normal to the mainstream flow direction were obtained from a sequence of 1,000 frames of instantaneous PIV measurement results. The uncertainty level for the PIV measurements is estimated to be within $3 \%$ for the instantaneous velocity vectors, while the uncertainties for the measured ensemble-averaged flow quantities such as vorticity distributions are about $10 \%$.

\section{Measurement Results and Discussions}

\subsection{Verification of measured film cooling effectiveness by using PSP technique.}

While a number of experimental studies have been conducted in recent years by using PSP technique to achieve quantitative measurements of adiabatic film cooling effectiveness, PSP technique is still a relatively new technique for film cooling studies, in comparison to conventional temperature-based measurement methods. It is necessary to validate the reliability of the PSP measurements in order to make PSP technique as an effective experimental tool for turbine blade film cooling studies. With this in mind, a comparative study was conducted to provide a quantitative comparison of the measured film cooling effectiveness by using PSP technique against those derived from temperature-based measurements of the previous studies [32-34] under same or comparable test conditions. It is worth noting that, the spanwise periodicity of the coolant streams were first checked in the present study, and very similar coolant coverages were observed for the all the coolant injection holes. Therefore, only the measurement results behind the coolant injection hole in the middle of the test plate are shown here for conciseness. It should also be noted that, the centerline cooling effectiveness profiles given in the present study refer to the measured film cooling effectiveness values along the centerline of the coolant injection hole, while the laterally-averaged cooling effectiveness is determined by averaging the measured film cooing effectiveness along the spanwise direction over one full period of the hole spacing (i.e., in the region of $-1.5 \leq Z / D \leq 1.5$ ) behind the coolant injection hole. As shown in Fig. 5, the measured centerline cooling effectiveness profile of the present study was found to agree with those of the previous studies with temperature-based measurements well over the entire compared region (i.e., up to the downstream location of $\mathrm{X} / \mathrm{D}>30$ ). For the laterally-averaged film cooling effectiveness, while the measurement results of the present study was still found to be consistent with the archived measurement data of the previous studies reasonably well in general, the PSP based measurement results of the present study seems to be slightly greater than those of the temperature-based measurements, especially in the region near the exit of the coolant injection hole (i.e., $\mathrm{X} / \mathrm{D}<7.0$ ). 


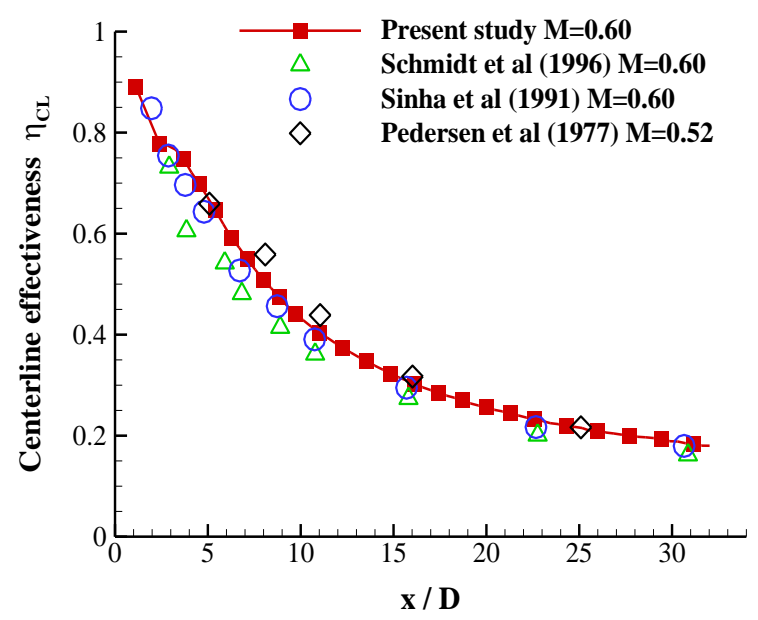

(a). Centerline film cooling effectiveness

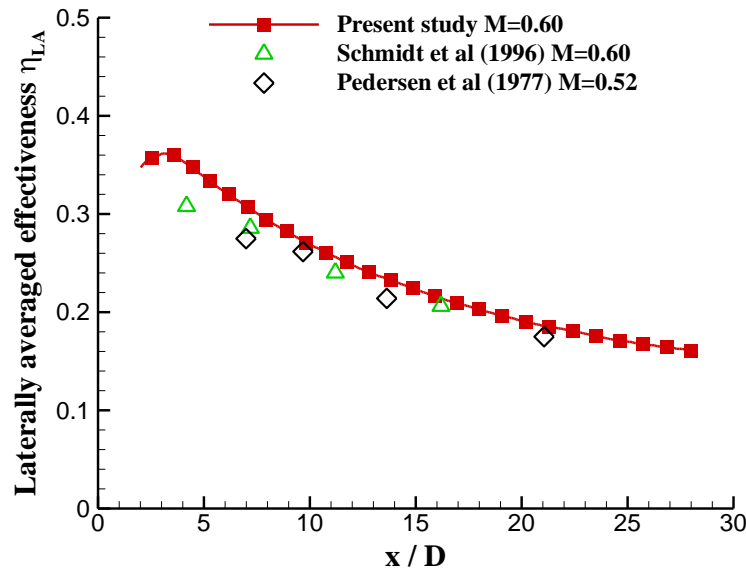

(b). Laterally-averaged effectiveness

Figure 5. Comparison of the measured film cooling effectiveness of the present study with those of the previous studies at the test conditions of $M=0.60, D R=1.53$, and $P / D=3$.

\subsection{Effects of the blowing ratio on the film cooling effectiveness behind BDSR}

Figure 6 shows the measured film cooling effectiveness distributions over the surface of the test plate for the new film cooling design with BDSR at different blowing ratios (i.e., mass flux ratios), in comparison with those of the baseline case (i.e., the case without BDSR). As shown clearly in Fig. 6 (a), while the effectiveness of the film cooling over the test plate was found to be much better for the test cases with BDSR than that of the baseline case at relatively large blowing ratios (i.e., at $M>0.85$ ), the existence of the BDSR has very limited or even detrimental effects on the film cooling effectiveness at relatively low blowing ratios (i.e., at $M<0.85$ ). As revealed clearly in Johnson et al.[23], at relatively low blowing ratios (e.g., $M \approx 0.4$ ), the coolant streams out of the coolant injection holes would remain attached to the surface of the test plate to provide a nice protection to the surface of interest. As shown in Fig. 6, the existence of the BDSR would actually cause slightly detrimental effects on the film cooling effectiveness over the test plate at the relatively low blowing ratio of $M=0.40$, especially in the region near to the exits of the coolant injection holes. However, at relatively high blowing ratio of $M \geq 0.85$, the regions with high cooling effectiveness values were found to become much narrower and shorter for the baseline case, indicating the high momentum coolant jet streams separated from the surface of plate. Under such conditions, the existence of the BDSR was found to cause much better coverage of the coolant stream over the surface of the interest in both spanwise and stream-wise directions, in comparison with those of the baseline case without BDSR. It suggests that the existence of the BDSR is very beneficial to improve film cooling effectiveness over the surface of interest, even with the same amount of coolant fluid exhausted from the same coolant injection holes.

The effects of the BDSR on the film cooling effectiveness over the surface of interest were revealed more quantitatively from the quantitative comparison of the centerline and laterally-averaged cooling effectiveness profiles between the test cases with the BDSR and the baseline case, which are shown in Fig.6(b) and Fig. 6(c) at relatively low blowing ratios (i.e., $\mathrm{M} \leq 0.85$ ) and relatively high blowing ratios (i.e., $M>0.85$ ), respectively. It can be seen clearly that, at the relatively low blowing ratio of $M=0.40$, the absolute values of the measured cooling effectiveness (i.e., for both centerline and laterally-averaged profiles) for the test cases with BDSR were found to be slightly lower than those of the baseline case, which is consistent with the spatial contour distributions shown in Fig. 6(a). The film cooling effectiveness for the configuration with BDSR was found to start to exceed that of the baseline case as the blowing ratio increases to $M=0.85$. As the blowing ratio becoming greater than 0.85 (i.e., $M>0.85$ ), the film cooling performance for the configuration with BDSR was found to completely surpass the baseline 
case with an augmentation of $40 \% \sim 150 \%$ in the laterally-averaged cooling effectiveness values. Such measurement results indicate that the BDSR would significantly improve the performance of film cooling over the surface of interests, especially for the lateral-averaged cooling effectiveness, at relatively high blowing ratios.
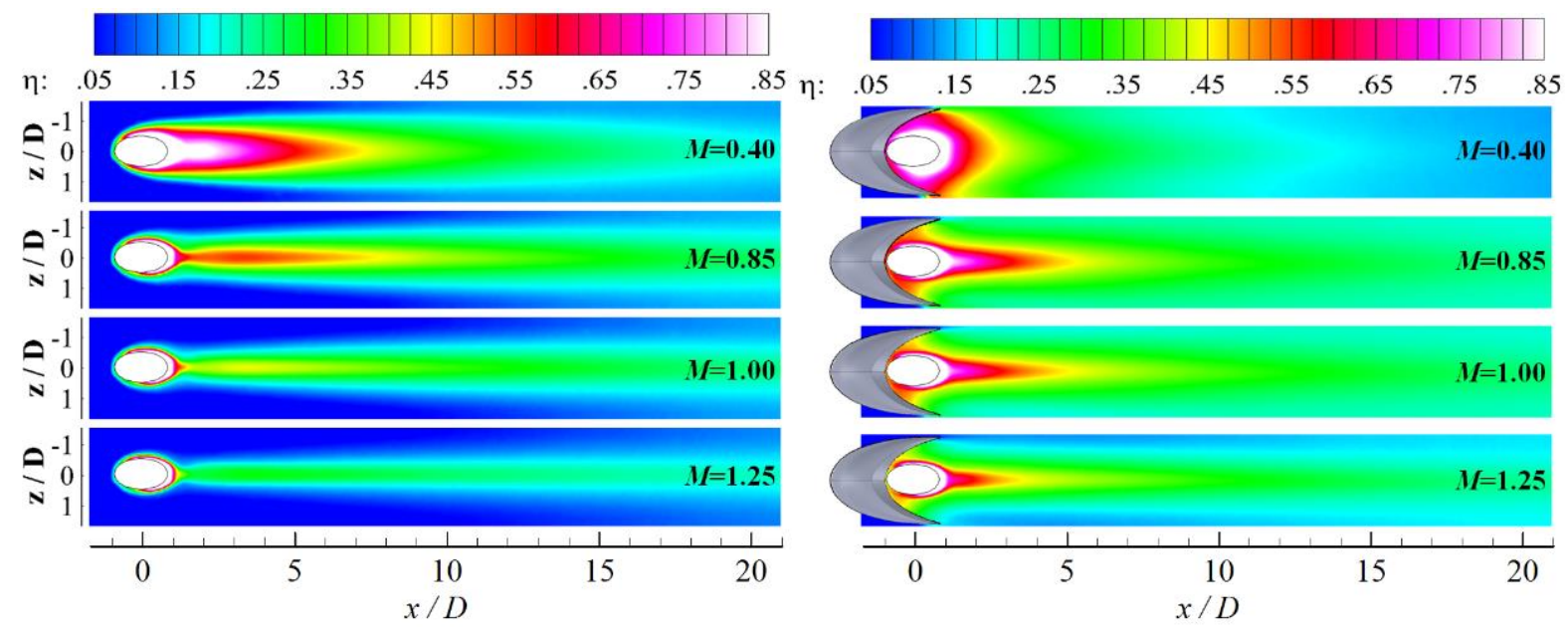

(a). Cooling effectiveness distributions for the baseline case (right) and the configuration with BDSR (left)
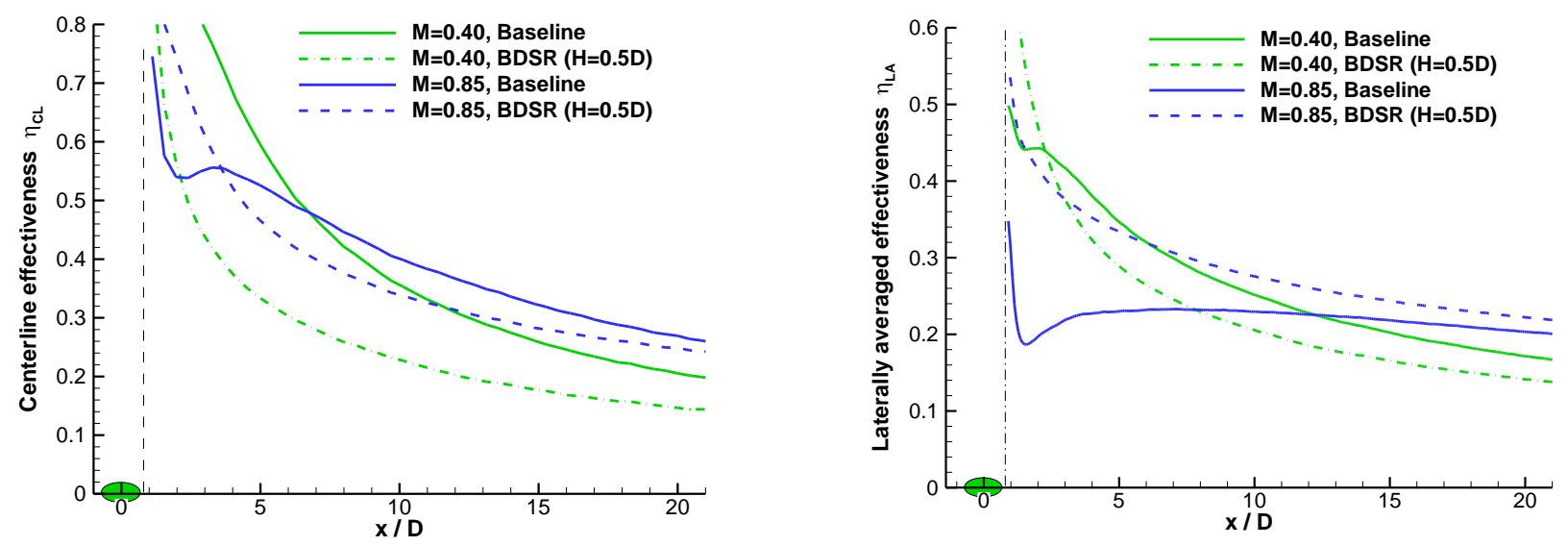

(b). The centerline and laterally-averaged cooling effectiveness profiles at $M=0.40$ and 0.85
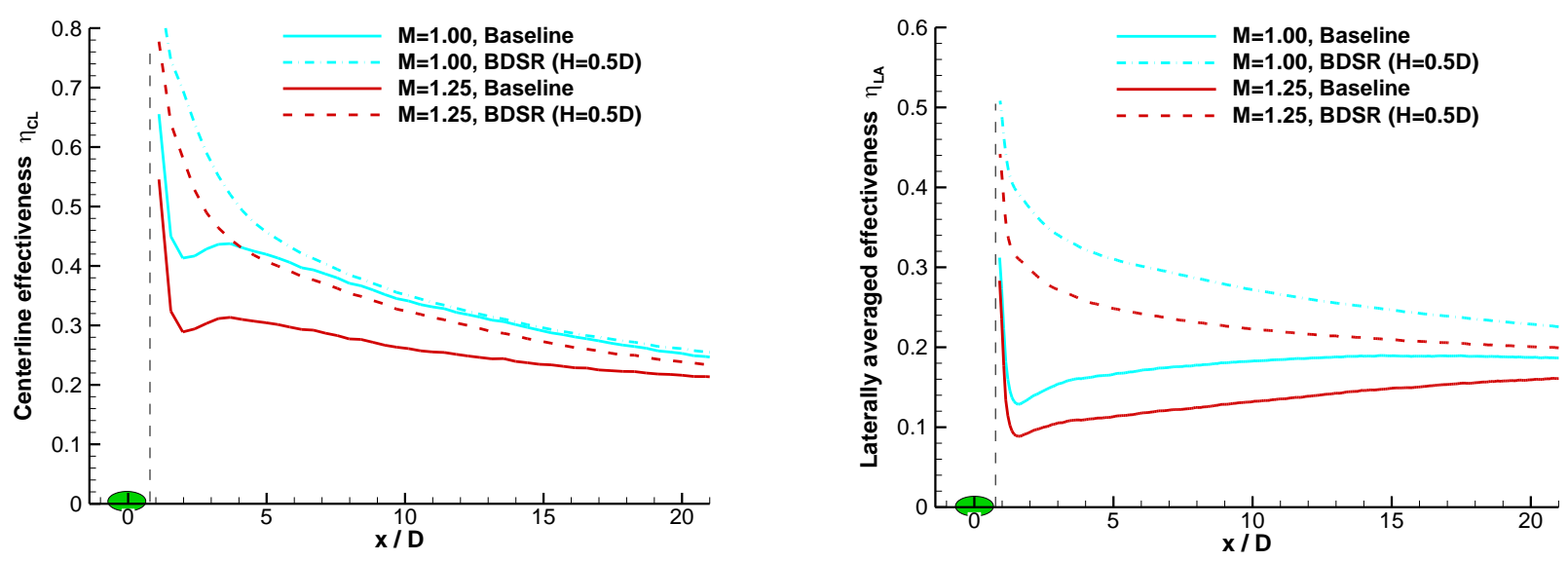

(c). The measured centerline and laterally-averaged cooling effectiveness at $M=1.00$ and 1.25

Figure 6. Comparison of the measured film cooling effectiveness between the new configuration with BDSR $(H=0.5 D, L=-0.9 D)$ and the baseline case at various blowing ratios. 


\subsection{PIV measurements for the test cases with BDSR and baseline cases}

While the measured film cooling effectiveness results given above reveals clearly that the existence of the BDSR would improve the performance of film cooling greatly at relatively high blowing ratios (i.e., $M>0.85$ ), it is highly desirable to elucidate the underlying physics pertinent to the improved film cooling effectiveness. With this in mind, a high-resolution PIV system was used to conduct detailed flow field measurements to quantify the dynamic mixing between the mainstream and coolant stream flows over the test plate. Figure 7 and Fig. 8 shows the PIV measurement results in the streamwise plane (i.e., $X-Y$ plane as shown in Fig. 4) for the baseline case (left column) and the new configuration with BDSRs (right column) at the blowing ratios of $M=0.40$ and $M=1.00$, respectively. The instantaneous vorticity distributions shown in Fig. 7(a) and Fig. 8(a) reveal that, as exhausted from the coolant injection holes, the coolant jet streams would mix with the mainstream flows intensively over the test plate. Due to the Kelvin-Helmholtz instabilities in the shear layers between the coolant streams and mainstream flows, unsteady vortex structures were found to be generated and shed periodically in the shear layers over the test plate. As expected, the appearance of the vortex structures were found to be more closely to surface of the test plate at relatively low blowing ratio of $\mathrm{M}=0.4$, in comparison that at relatively high blowing ratio of $M=1.00$.

As shown clearly in Fig. 7 (b), the coolant streams exhausted from the coolant injection holes would stay attached to the surface of the test plate in general at the relatively low blowing ratio of $M=0.40$ for both the baseline case and the test case with BDSR. After a careful inspection of the flow features for the two compared cases, the streamlines exhausted from the coolant injection hole for the baseline case seem to be able to stay slightly closer to the surface of the test plate, indicating a slightly better film cooling protection to the surface of interest at the low blowing ratio of $M=0.40$, in comparison to the case with BDSR. The experimental observation on the flow pattern is found to be in good agreement with the measured film cooling effectiveness distributions described above.
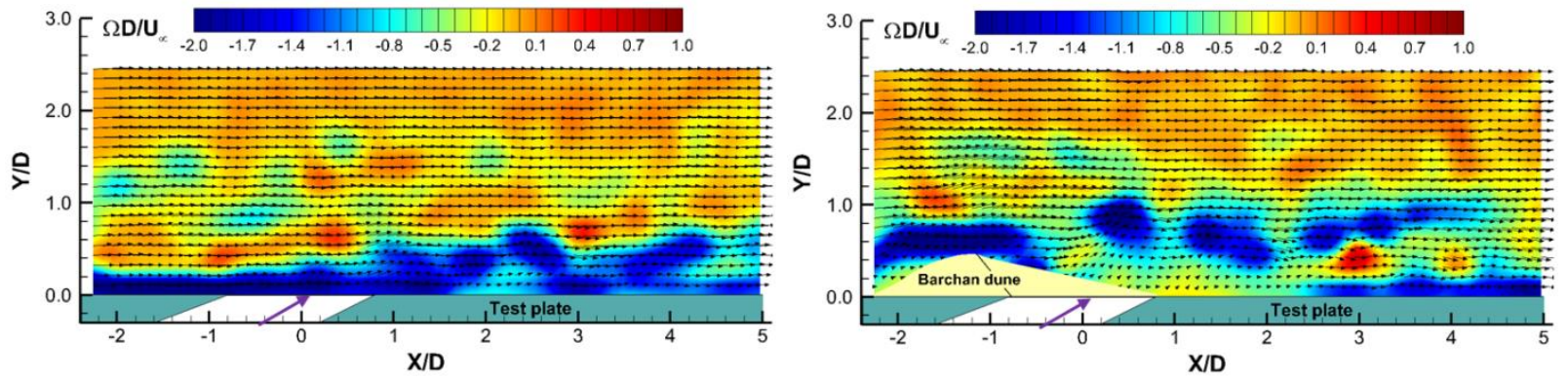

(a) Instantaneous vorticity distributions
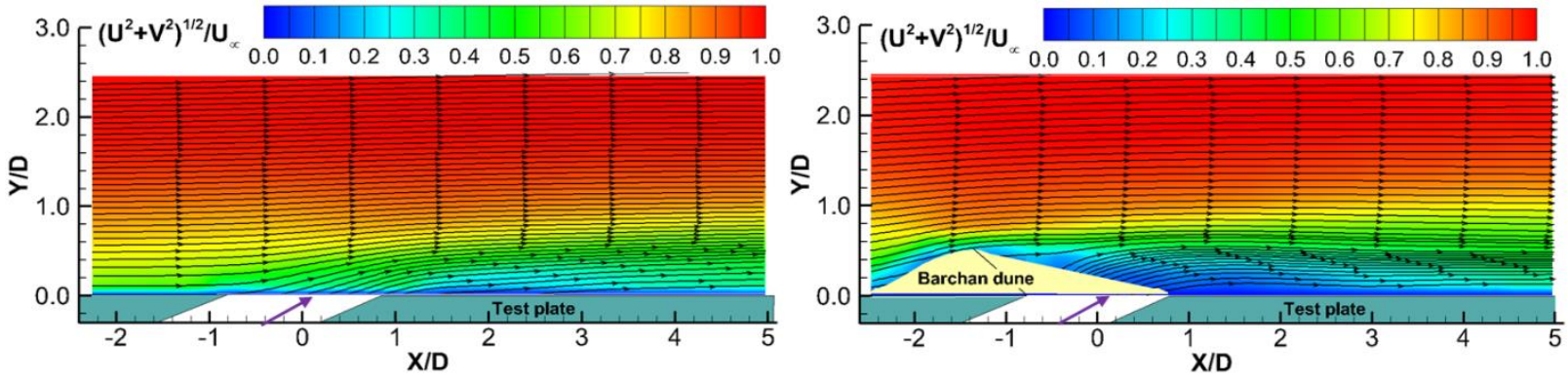

(b) Ensemble-averaged streamlines and velocity fields

Figure 7. PIV measurement results for the baseline case (left column) and the case with BDSR (right column) at a relatively low blowing ratio of $M=0.40$. 
However, the situation was found to change completely at the relatively high blowing ratio of $M=1.00$. As revealed clearly in Fig. 8, after exhausted from the coolant injection hole, the streamlines of the coolant jet stream for the baseline case were found to separate from the surface of the test plate and penetrate into the mainstream flow at the blowing ratio of $M=1.00$, causing poor film cooling protection over the surface of interest. While a similar trend was also observed for the case with BDSR, the separation and penetration of the coolant jet stream were found to become much mild due to the existence of the BDSR on the test plate. A more careful inspection of the flow features around the BDSR reveals that a downwash flow was found to be generated at the top of the BDSR to suppress the take-off of the coolant jet stream from the surface of the test plate. This is believed to be closely related to the suction effect induced by the BDSR, which will be discussed further in the later section. As a result, the film cooling effectiveness over the surface of interest was found to become higher for the BDSR case than that of the baseline case at the blowing ratio of $M=1.0$, which was revealed quantitatively from the measured distributions of the film cooling effectiveness given in Fig. 6.
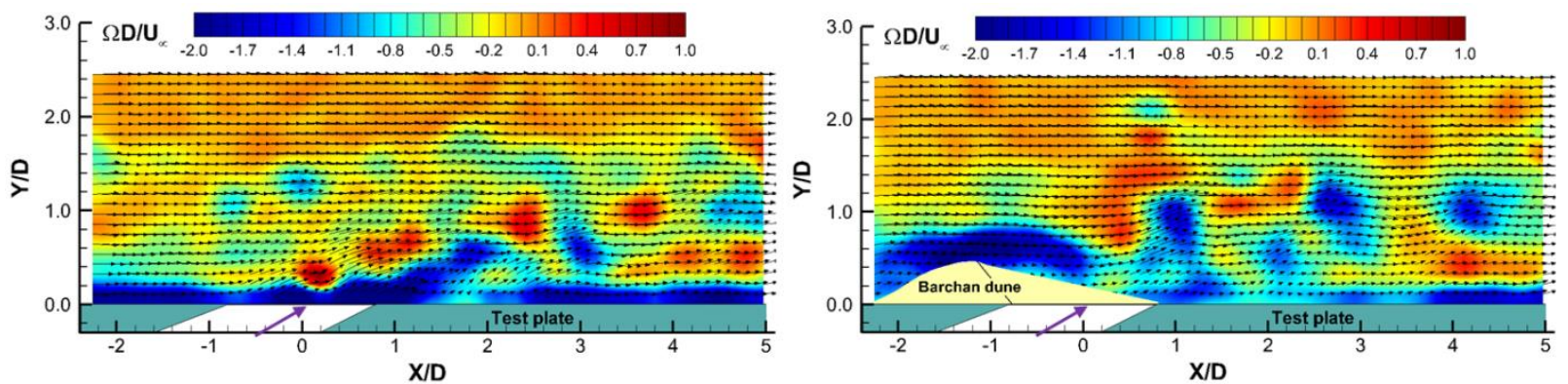

(a) Instantaneous vorticity field
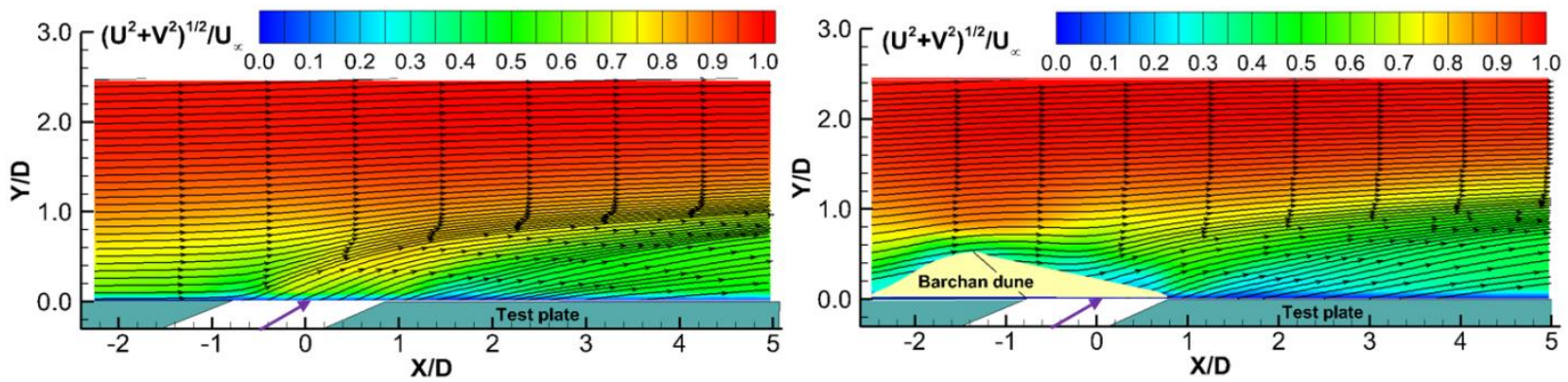

(b) Ensemble-averaged velocity field

Figure 8. PIV measurement results for the baseline case (left column) and the case with BDSR (right column) at a relatively high blowing ratio of $M=1.00$.

\subsection{Stereoscopic PIV measurements for the BDSR case and the baseline case.}

As described above, film cooling, fundamentally, is a jet-in-cross-flow (JICF) problem. The dominated flow features for a JICF include shear-layer-related vortices formed at the jet leading edge, horseshoe vortices around the jet, counter-rotating-vortex (CRV) pair in the jet, and wake vortices downstream of the jet [3]. As shown in the PIV measurement results given in Fig. 8, the coolant jet stream tends to separate from the surface of interest and penetrate into the mainstream flow due to the induction from the strong CRV at relatively high blowing ratios, which leads to poor film cooling performance over the surface of interest. As demonstrated by the work of Zaman et al. [5] and Shinn et al. [6], a promising method to improve film cooling effectiveness is to reduce the detrimental effects of the vortex induction of the CRV by introducing a pair of anti-CRV to weaken the strength of CRV to make the coolant jet stream stay attached to the surface of interest. 
In the present study, a stereoscopic PIV (SPIV) system was also used to reveal the generation and evolution of various streamwise vortex structures in the coolant jet stream for the test case with BDSR mounted on the test plate, in comparison with those of the baseline case in order to gain further insight into the inherent mechanism pertinent to the improved film cooling performance due to the effects of the BDSR. Figure 9 shows some typical SPIV measurement results (i.e., ensemble-averaged measurement results) in the cross planes normal to the flow direction of the mainstream (i.e., within Y-Z plane). As shown clearly in Fig. 9(a), a pair of streamwise counter-rotating vortices (CRV) were found to be generated behind the coolant injection hole as the salient flow structure for the baseline case, where the near-wall coolant flow was found to eject away from the surface of the test plate by an upwash flow formed between the CRV pair.

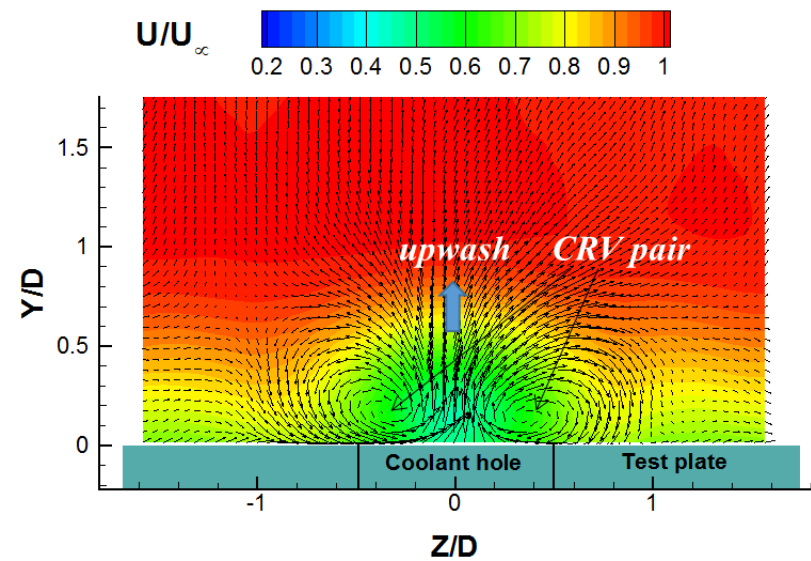

(a). Baseline case in the $X / D=3.0$ cross plane

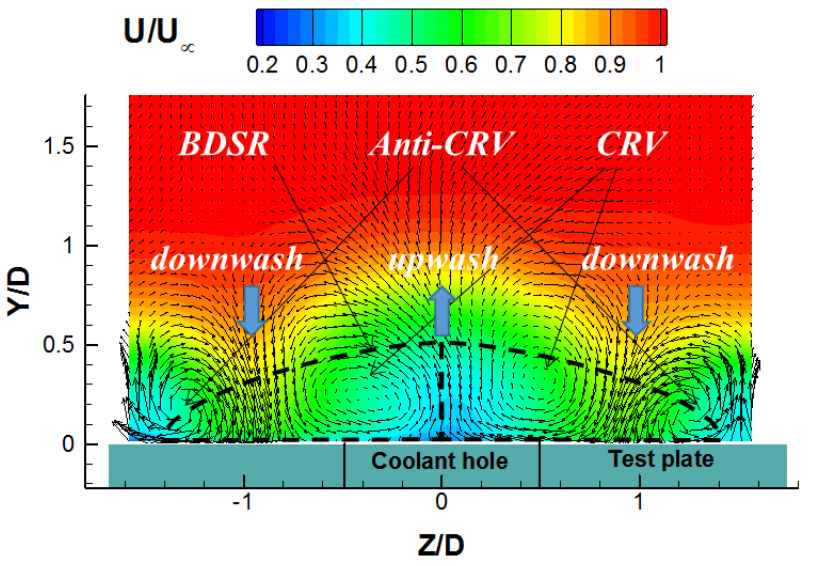

(b). BDSR case in the $X / D=3.0$ cross plane.

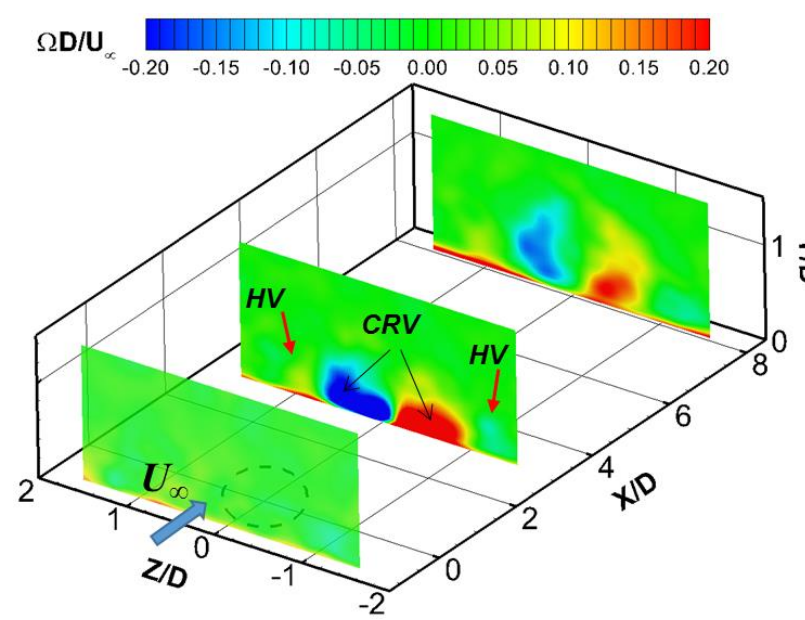

(c). Evolution of the vortx structures for the baseline case

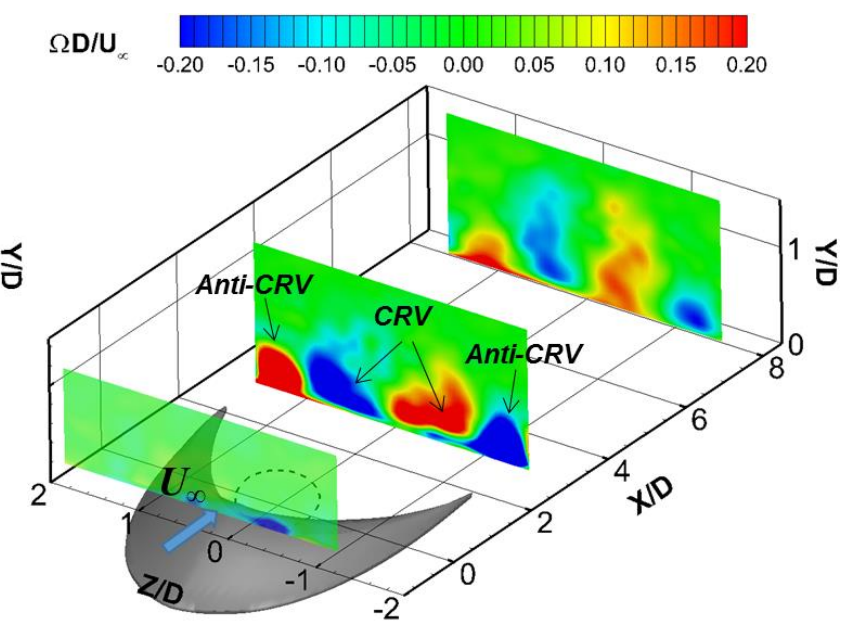

(d).Evolution of the vortx structures for the BDSR case

Figure 9. Stereoscopic PIV measurement results for the baseline and the test case with BDSR

The SPIV measurements given in Fig. 9(b) reveal clearly that, with a BDSR mounted in front of the coolant injection hole, an additional pair of streamwise counter-rotating vortices would be generated behind the BDSR, besides the CRVs induced by the coolant jet stream. The existence of the counterrotating streamwise vortex pair behind the two horns of a Barchan dune was also observed by Zheng et al. [35] and Omidyeganeh et al. [36]. Since the rotation directions of the streamwise vortices induced by the BDSR were found to be opposite to those of the CRVs in the coolant jet stream, they are referred as anti- 
CRVs. The anti-CRVs could countervail the detrimental effects of the CRVs in the coolant jet stream. Consequently, the seperation of the coolant jet stream from the surface of the test plate would be postponed due to the existance of the BDSR, in comparison to the baseline case. As a result, as shown clearly in Fig. 8(b), downwash flows were found to be generated in the regions between the streamwise vortices, which would push the lifted coolant jet streams back to the surface of interest for a better flim cooling protection.

In comparisn with the baseline case, the existance of the BDSR would also make a faster spanwise spreading of the coolant stream over the test surface. The distance between the CRVs in the coolant jet stream were found to be elongated in the lateral direction due to the existance of the BDSR, in comparison to the baseline cas. The flow features would result in a wider coverage of the coolant stream over the surface of interest, as revealed clearly from the measured film cooling effectiveness distributions given in Fig. 6. It can also be seen that, a relative low velocity region (i.e., as indicated as th region in light blue color in the Fig. 9(b)) was found to exist in the near wall region for the test case with BDSR, which indicates a "quiet" wake flow behind the BDSR. Such flow features were also revealed clearly in the 2-D PIV measurements along the streamwise plane, as shown in Fig. 8.

Figure 9 (c) and Fig. 9(d) show the evolution of the ensemble-averaged streamwise vorticity distributions for the two compared cases at three typical streamwise locations (i.e., at the locations of $X / D=-1.3,3.0$, and 8.0, respectively). It can be seen clearly that, the flow features for the baseline case were characterized with a pair of large-scale streamwise CRVs behind the coolant injection hole and a pair of horeshoe vorticies $(H V)$ with much lower vortex strength at the two sides. As reported in many previous studies on JICF [3,37-39], these streawise vortex structures are the dominating flow features of an inclined jet flow. As shown clearly in Fig. 9(c), the streawise vortex structures would be dissipated gradually due to the viscois effects, as they travel downstream. For the test case with BDSR, while the features of the vortex structures were found to be similar as those of the baseline case, the strength of the anti-CRVs generated behind the BDSR were found to be much stronger than that of the $H V s$ for the baseline case. The anti-CRV was found to interact intensively with the CRVs in the JICF, which would suppress the lift-up of the coolant jet stream from the surface of interest.

Based on the SPIV measurements as those given in Fig. 9, a sketch of the flow features behind the BDSR was conjectured, which is given in Fig. 10. The diagram vividly illustrates the unique flow features behind the BDSR, which can be used to reveal the underlying physics pertinent to the improved film cooling performance for the case with BDSR at relatively high blowing ratios. As shown in the diagram, the vortex structures behind the BDSR are highly three dimensional, featured by two pairs of large-scale vortices. The first pair of vortices were found to be in the horizontal plane (i.e., $X-Z$ plane) behind the BDSR, as reported by Christensen et al.[15]. This pair of vortices could generate continuous suction along the ridges of the BDSR to make the coolant stream to spread out more widely and uniformly over the surface of the test plate. The second pair of the vortices are the streamwise anti-CRVs generated behind the horns of the dune, which are in the vertical plane normal to the flow direction of the oncoming mainstream flow (i.e., within $Y-Z$ plane). The streamwise anti-CRVs were found to counteract the detrimental effects of the CRVs in the coolant jet stream to generate downwash flows to bring the separated coolant stream back to the surface of interest. As a result, the coolant jet stream, after exhuasted from the coolant injection hole, would more likely to stay attached over the surface of interst and provide a better coverage over the test plate along the spanwise direction. In summary, the augmentation of the film cooling effectiveness for the test case with BDSR as revaled in Fig. 6 is suggested to be caused by the downwash effects induced by the streamwise anti-CRVs generated behind the BDSR along with the dynamic interactions among various complex vortex structures generated in the wake flow of the BDSR. 


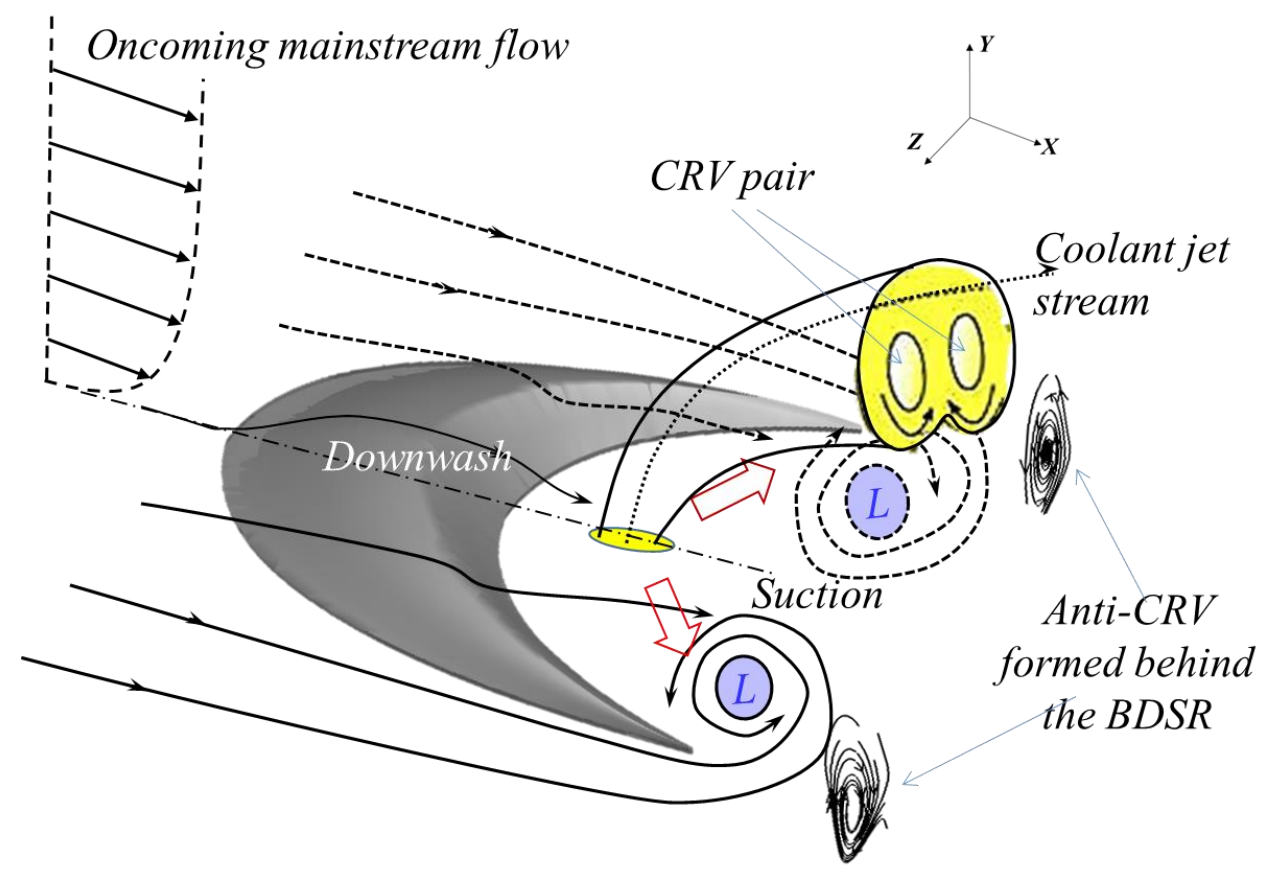

Figure 10. Schematic of the vortex structures behind the BDSR

\subsection{The effects of BDSR location in relation to the coolant injection hole on the film cooling performance}

The film cooling for a gas turbine blade is usually achieved by injecting coolant flow from arrays of coolant injection holes. In such a setting, the DBSR placed upstream of a coolant injection hole (i.e., the configuration as shown in Fig. 6) could be located at the downstream of the upstream coolant injection holes. While the measurement results given in Fig. 6 indicate that the existence of the BDSR at the upstream of a coolant injection hole can enhance the film cooling effectiveness substantially at relatively high blowing ratios, it is also meaningful to examine the effects of the BDSR location in relation to the coolant injection hole on the film cooling performance in order to explore/optimize design paradigms for improved film cooling effectiveness with arrays of coolant injection holes. Figure 11 shows the measured film cooling effectiveness as a function of the distance $(L)$ between the BDSR and the coolant injection hole at the blowing ratio of $M=0.85$ (left column) and $M=1.25$ (right column), respectively. It is apparent that there are great differences in the measured film cooling effectiveness for the settings with the DBSR being placed either upstream or downstream of the coolant injection hole. With the BDSR located at the upstream of the coolant injection hole (i.e., the distance $L$ becoming negative), the film cooling performance for the test case with $L=-0.9 D$ was found to be better than that of the test case with $L=-3.0 D$ at both $M=0.85$ and $M=1.25$. It is because that the downwash flows and suction effects induced by the BDSR would become stronger as the BDSR was placed more closely to the coolant injection hole.

However, for the configuration with the BDSR located at the downstream of the coolant injection hole, the test case with $L=3.4 D$ was found to exhibit a higher film cooling effectiveness than that of $L=2.7 \mathrm{D}$ case. It suggests that the film cooling effectiveness would be slightly better if the BDSR was placed at a further downstream location away from the coolant injection hole. By analyzing the film cooling effectiveness for all the compared cases, the test case with $L=-0.9 D$ (i.e., BDSR located at the upstream of the coolant injection hole with a smaller distance of $L / D=0.9 D$ ) was found to display the highest film cooling effectiveness at relative high blowing ratios, followed closely by the test case of $L=3.4 \mathrm{D}$ (i.e., with the BDSR located at a further downstream location away from the coolant injection hole). 


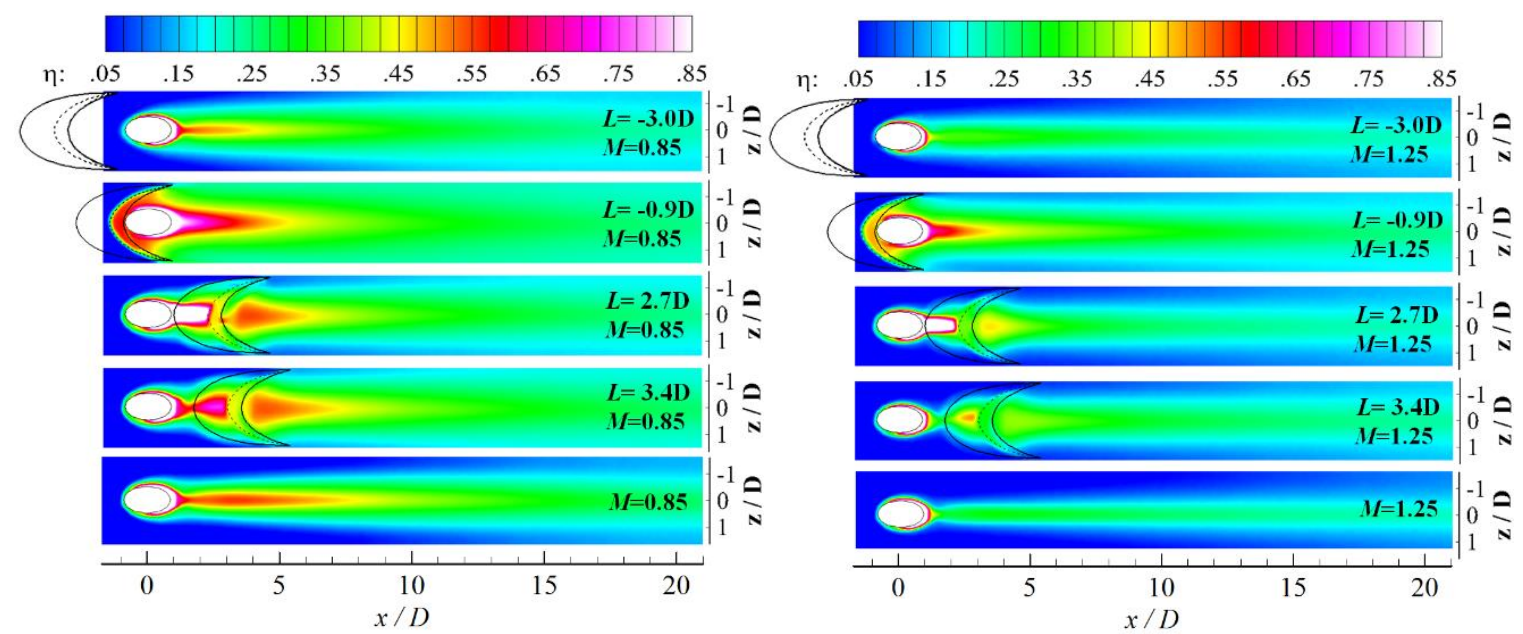

(a). Cooling effectiveness distributions at the blowing ratios of $M=0.85$ (right) and $M=1.25$ (left)
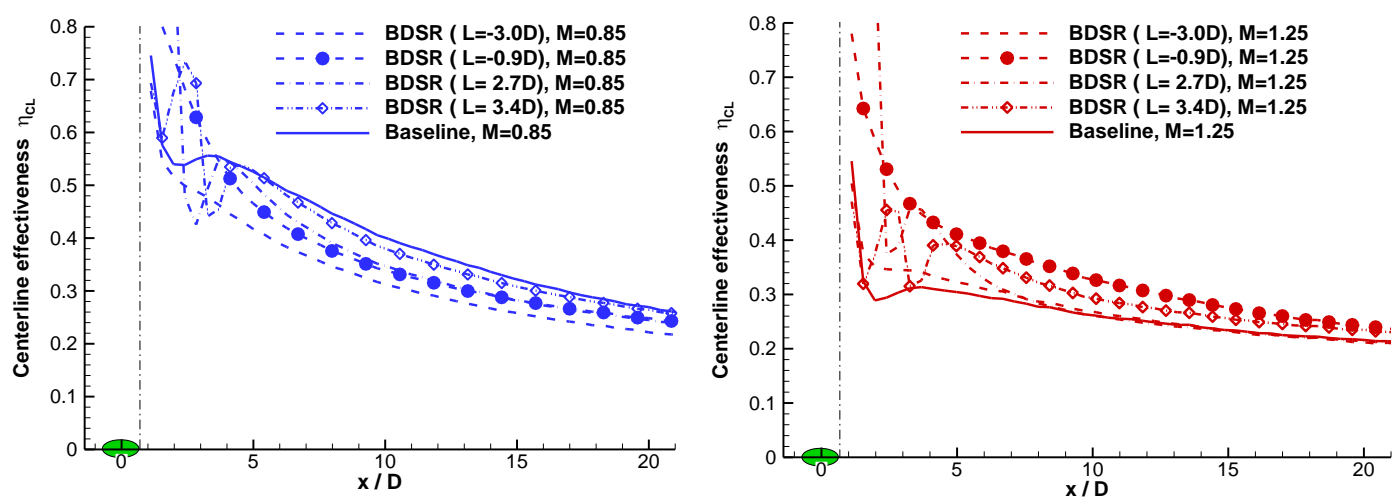

(b). Centerline cooling effectiveness profiles at $\mathrm{M}=0.85$ (right) and $\mathrm{M}=1.25$ (left)
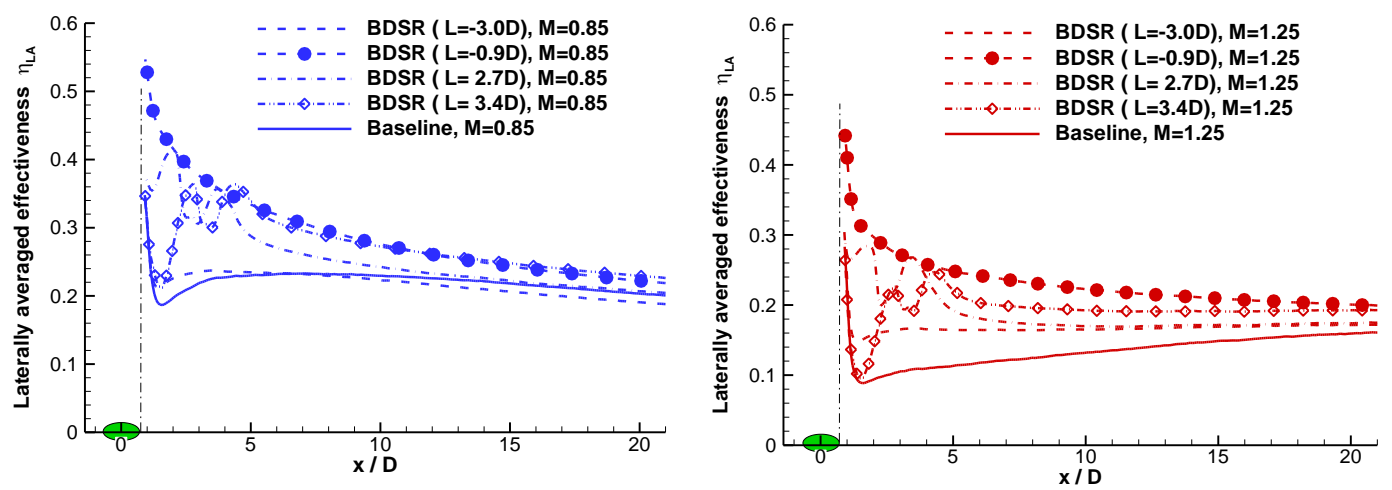

(c). Laterally-averaged cooling effectiveness profiles at $\mathrm{M}=0.85$ (right) and $\mathrm{M}=1.25$ (left)

Figure 11. Measured film cooling effectiveness as a function of the distance between the BDSR and the coolant injection hole at the blowing ratios of $M=0.85$ (left) and $M=1.25$ (right).

Figure 12 shows the PIV measurement results in the streamwise plane with the BDSR located at either upstream (left column, with $L=-0.9 \mathrm{D}$ ) or downstream (right column, with $L=3.4 \mathrm{D}$ ) of the coolant injection hole at the blowing ratio of $M=1.0$ (i.e., the corresponding velocity ratio being $0.66, V_{c} / V_{\infty}$ $=0.66$ ). As described above, since a shorter distance between the BDSR and the downstream coolant injection hole would result in a faster laterally expending and a stronger downwash effects induced by the 
BDSR, the film cooling performance over the surface of interest was found to increase greatly when the BDSR was placed immediately upstream of the coolant injection hole (i.e., for the test case with $L=-$ 0.9D). As shown clearly in Fig. 12(a), due to the downwash effects induced by the BDSR, the streamlines of the mainstream flow were found to bend downward slightly as passing over the ridge of the BDSR. Consequently, the coolant jet streams were found be confined in the region closer to the surface of the test plate, in comparison with the case without the BDSR.

As shown in Fig.12(b), for the case with the BDSR placed at the downstream of coolant injection hole (i.e., $L=3.4 D$ case), due to the Coanda effect (i.e., the phenomena in which a jet flow tends to attach itself to a nearby surface and remains attached even when the surface curves away from the initial jet direction), the separated coolant jet stream was found to stay attached to the surface of the BDSR. As a result, the separated coolant stream would be able to re-attach to the surface of interest at the downstream locations behind the BDSR to provide a better film cooling performance, as shown quantitatively in the measured film cooling effectiveness distributions given in Fig. 10.

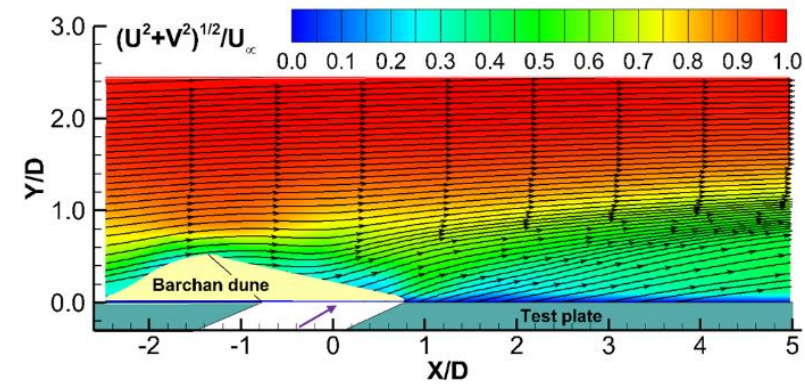

(a). BDSR at the upstream of the coolant hole

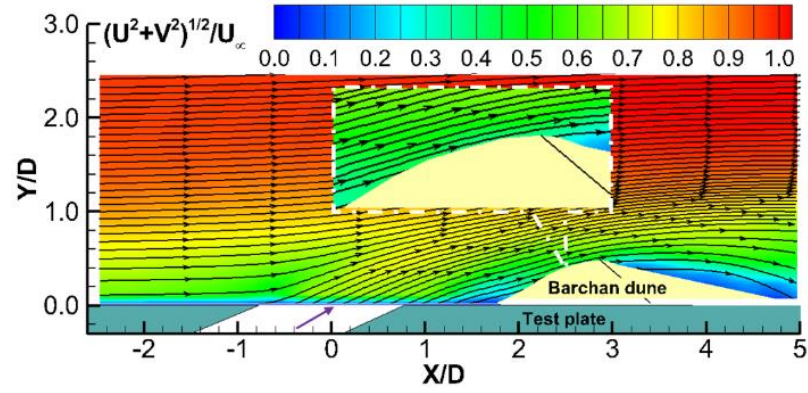

(b). BDRS at the downstream of the coolant hole

\section{Figure 12. PIV measurement results with BSRS located at either upstream (i.e., $L=-0.9 D$ ) or downstream (i.e., $L=3.4 \mathrm{D}$ ) of coolant injection hole at the blowing ratio of $M=1.0$.}

\subsection{The effect of BDSR height on the film cooling performance.}

Since the configurations with $L=-0.9 D$ and $L=3.4 D$ were found to exhibit better film cooling performance in comparison with the other test cases, these two confications were chosen for further investigation to examine the effects of BDSR heigh on the film cooling performance over the surface of interest. It should be noted that, all of the measurement results given above were obtained with the height of the BDSR being 0.5D (i.e., $H=0.5 D$ ). In order to evaluate the effects of BDSR heigh on the film cooling performance, a new set of BDSRs with a smaller height of $H=0.3 D$ were designed and manufactured for the comparative study. Figure 13 shows some typical measurements results in terms of the centerline and laterally-averaged cooling effectiveness profiles. As shown clearly in Fig. 13 (a), with the BDSR placed upstream of the coolant injection hole (i.e., the configuration with $L=-0.9 \mathrm{D}$ ), the test case with a higher BDSR (i.e., $H=0.5 \mathrm{D}$ case) was found to exhibit an improved film cooling performance than that with a smaller BDSR height (i.e., $H=0.3 D$ case) at both the blowing ratios of $M=0.85$ and $M=1.25$. This is believed to be caused by the stronger downwash and suction effects with a higher BDSR. For the configuration with the BDSR placed at the downstream of the of the coolant injection hole (i.e., the configuration with $L=3.4 D$ ), the film cooling effectiveness of the test case with a smaller BDSR height (i.e., $H=0.3 \mathrm{D}$ ) was found to be slightly better than that with greater BDSR height (i.e., $H=0.5 \mathrm{D}$ case).It suggests that the smaller DBSR could be a better choice for the configuration with the BDSR located at the downstream of the coolant injection hole. 

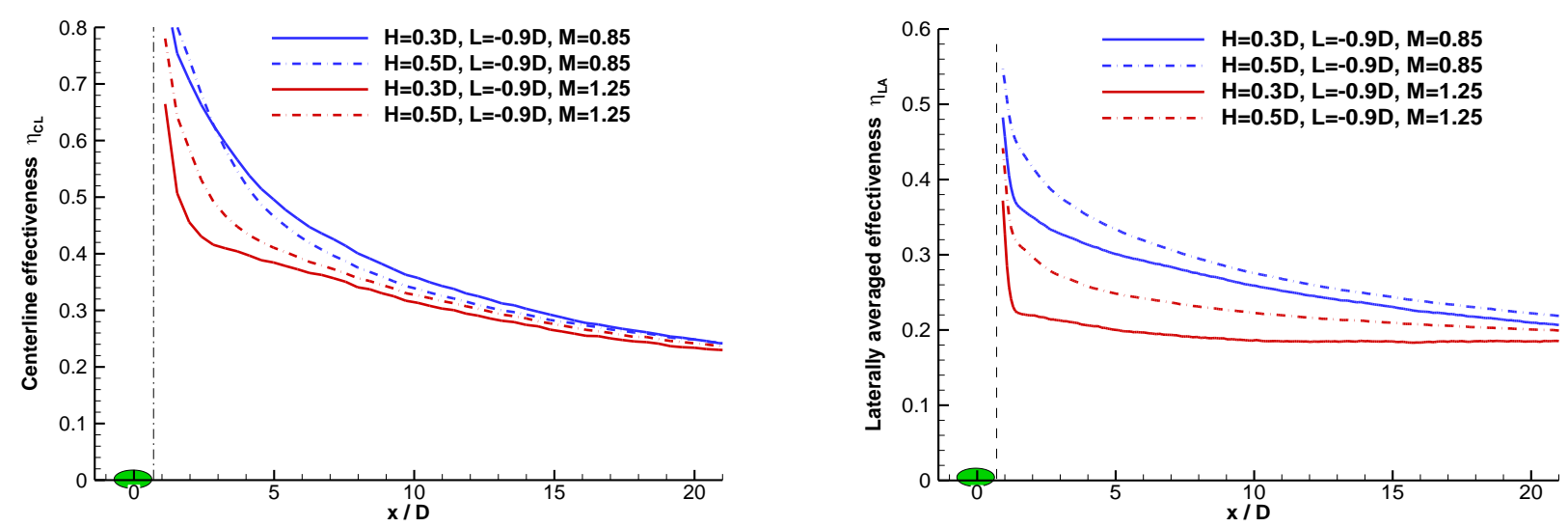

(a). Measured cooling effectiveness with the BDSR placed at the upstream of the coolant injection hole $(L=-0.9 D)$
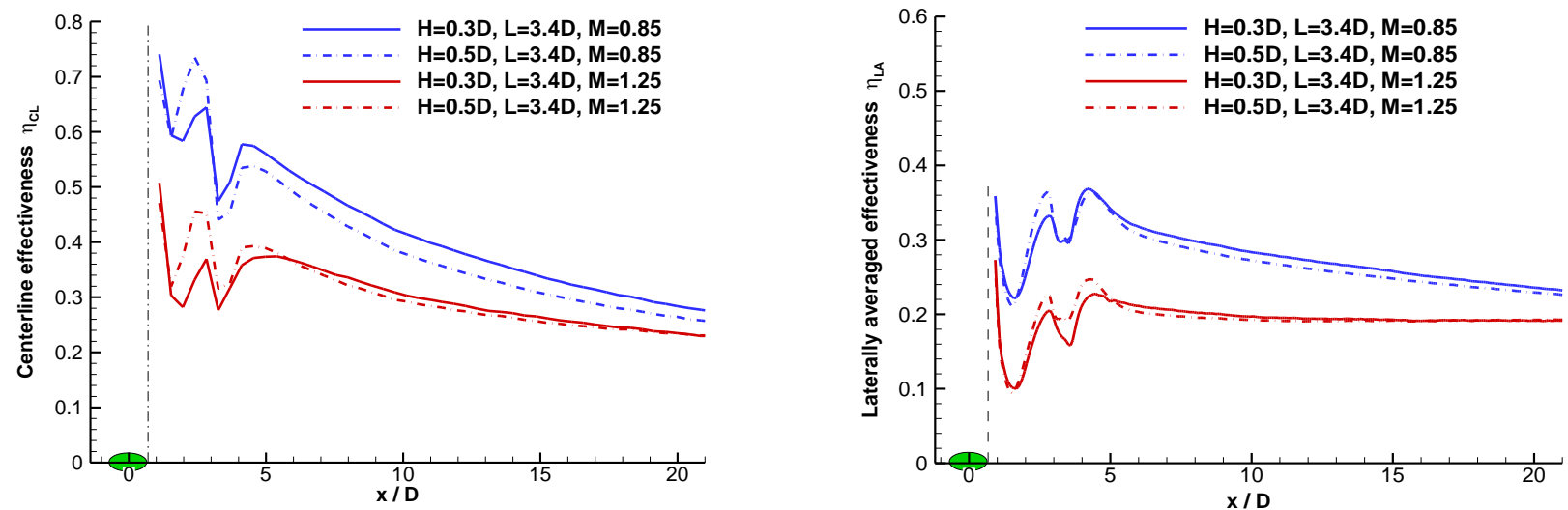

(b). Measured cooling effectiveness with the BDSR at the downstream of the coolant injection hole $(L=3.4 D)$

Figure 13. Effects of the height of the BDSR on the film cooling performance for the configurations with the BDSR at either upstream $(L=-0.9 D)$ or downstream $(L=3.4 D)$ of the coolant injection hole.

\subsection{Area-averaged film cooling effectiveness.}

In the present study, a parameter, named the area-averaged film cooling effectiveness, is introduced, to compare the overall performance of the film cooling effectiveness for the different test cases. The areaaveraged film cooling effectiveness, $\eta_{\text {area-cveraged }}$, is defined as $\eta_{\text {area-averaged }}=\frac{1}{A} \iint \eta_{x z} d A$, where $A$ is the area of the interest over the test plate (i.e., the region of $1 \leq X / D \leq 15$ and $-1.5 \leq Z / D \leq 1.5$ for the present study), and $\eta_{x z}$ is the corresponding cooling effectiveness at each location within the area of the interest. The standard deviation values of the measured film cooling effectiveness within the interest of area were also calculated in the present study. Obviously, a higher value of the area-averaged film cooling effectiveness, $\eta_{\text {area-averaged }}$, would indicate a better overall film cooling performance. A lower standard deviation value of the cooling effectiveness would represent a more uniform film cooling coverage over the area of interest.

Based on the experimental results described above, three representative configurations were selected for the comparative study in order to assess the overall film cooling performance. The three selected configurations include 1) the test case with the BDSR located at the upstream of the coolant injection with a higher BDSR height (i.e., the test case with $H=0.5 D$ and $L=-0.9 D$ ); 2).the test case with the BDSR located at the downstream of the coolant injection with a smaller BDSR height (i.e., the test case with $H=0.3 D$ and $L=3.4 D$ ); and 3) the baseline case without the BDSR. Figure 14 shows the area-averaged film cooling effectiveness and the corresponding standard deviations for the three selected configurations 
as a function of the blowing ratio. As shown clearly in Fig. 14, the BDSR case with $H=0.5 D$ and $L=-0.9 D$ was found to have the highest averaged film cooling effectiveness and lowest standard deviation at most of the bowling ratios. In comparison with the baseline case, an augmentation of 50\% 100\% was found to be achieved in the area-averaged film cooling effectiveness for the BDSR case with $H=0.5 D$ and $L=0.9 D$ at relatively high blowing ratios of $M>0.85$. The corresponding standard deviation values for the BDSR case with $H=0.5 D$ and $L=0.9 D$ were also found to be much smaller for most of the bowling ratios in comparison to the baseline case. All these results indicate that the film cooling configuration with DBSR can provide a better and more uniform film cooling over the surface of interest, especially for the test conditions that require high blowing ratios.

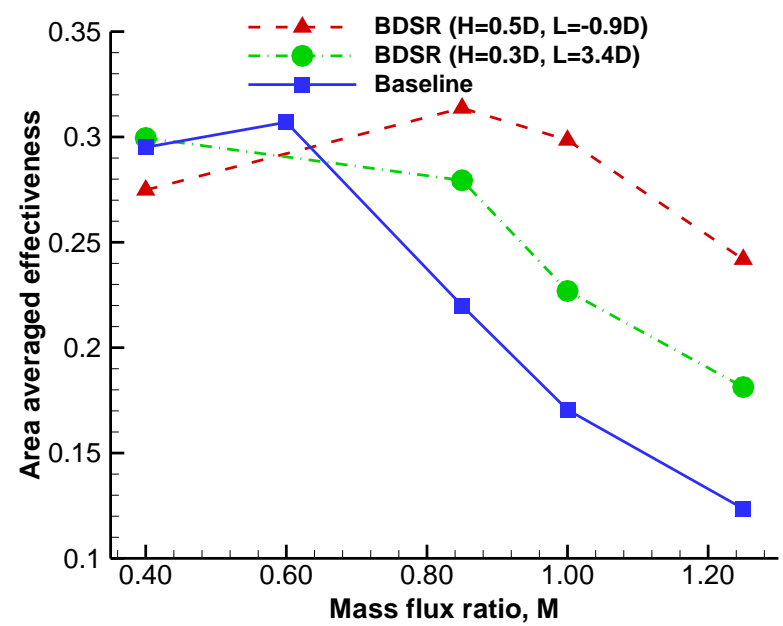

(a). area-averaged film cooling effectiveness

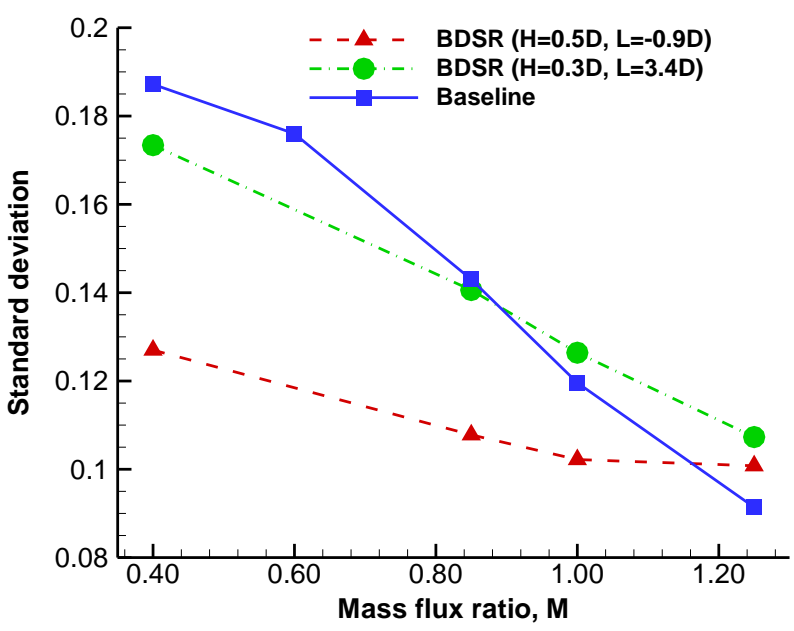

(b). Corresponding standard deviation values

Figure 14. The area-averaged film cooling effectiveness and the corresponding standard deviation values as a function of the mass flux ratio.

\section{CONCLUSION}

An experimental study was conducted to explore a novel film cooling design with a Barchan-duneshaped-ramp (BDSR) concept to improve the film cooling performance over the surface of a test plate with coolant streams injected from discrete circular holes at an injection angle of 35 degrees. As inspired by the unique shape of Barchan dunes commonly seen in deserts to prevent sand particles on the ground from being blown away by oncoming airflow, the BDSR concept was developed to generate a series of complex vortex structures in the wake behind the BDSR, which would be able to make the coolant stream flow stay more firmly over the surface of interest for improved film cooling effectiveness. The experimental study was conducted in a low-speed, open-circuit wind tunnel. While a Pressure Sensitive Paint (PSP) technique was used to map the adiabatic film cooling effectiveness on the surface of interest based on a mass-flux analog to traditional temperature-based cooling effectiveness measurements, a highresolution PIV system was used to conduct detailed flow field measurements in both streamwise and cross-flow planes to quantify the dynamic mixing process between the coolant jet streams and the mainstream flows over the test plate. The effects of the coolant blowing ratio (i.e., coolant-to-mainstream mass flux ratio, $M=0.40,0.85,1.00,1.25)$, the mounting location of the BDSRs in relation to the coolant injection holes (i.e., $L=-3.0 D,-0.9 D, 2.7 D, 3.4 D$ ), and the height of the BDSRs (i.e., $H=0.3 D, 0.5 D$ ) on the film cooling effectiveness over the surface of interest were examined in great detail based on PSP and PIV measurements.

The PSP measurement results revealed clearly that, with a same amount of the coolant streams exhausted from the injection holes, the film cooling effectiveness over the surface of interest can be 
enhanced greatly by mounting the BDSRs over the test plate, especially for the test cases with relatively high coolant blowing ratios (i.e., $M \geq 0.85$ ). More specifically, in comparison with those of the baseline case without BDSRs, the area-averaged film cooling effectiveness over the surface of interest was found to increase by $50 \% \sim 100 \%$ due to the existence of the BDSRs (i.e., the test case with $H=0.5 D, L=0.9 D$ ), as the coolant blowing ratios being $M \geq 0.85$. In addition to the higher overall film cooling effectiveness, the coverage of the coolant streams over the surface of the interest, especially along the spanwise direction, was also found to become much more uniform due to the existence of the BDSRs.

The PIV measurements reveal clearly that, the flow structures generated in the wake behind a BDSR are highly three dimensional, featured by two pairs of large-scale vortex structures. The first pair of vortices were found to be formed in the horizontal plane behind the dune, as reported by previous studies. This pair of vortex structures could generate continuous suction along the ridges of the BDSR to make the coolant streams to spread out more widely and uniformly over the surface of the test plate. The second pair of the vortices are the streamwise anti-CRVs generated behind the horns of the BDSR, which are in the vertical plane normal to the direction of the oncoming mainstream flow. The streamwise anti-CRVs were found to counteract the detrimental effects of the CRVs formed in the coolant jet stream to generate downwash flows to suck the separated coolant stream back to the surface of interest. As a result, the coolant jet stream would more likely to stay attached to the surface of interst and provide a better coverage over the test plate along the spanwise direction. In summary, the augmentation of the film cooling effectiveness due to the existance of the BDSR is believed to be closely related to the downwash effects induced by the streamwise anti-CRVs generated behind the BDSR along with the dynamic interactions among various complex vortex structures generated in the wake behind of the BDSR.

It should be noted that, while the findings of the present study demonstrated that the proposed BDSR concept can be used to augment the film cooling effectiveness over the surface of interest by generating a series of complex vortex structures over the test plate, more extensive investigations are still needed to elucidate the associated underlying physics and examine the relevant parameters, including discharge coefficients, heat transfer coefficients, the pressure losses, the compressibility of the gaseous flows, the geometry of the BDSR and the shape of the coolant injection holes, in order to explore/optimize design paradigms for better film cooling protection of turbine blades from harsh environments.

\section{Acknowledgment}

The technical assistances of Dr. Blake Johnson, Mr. Bill Richard, and Mr. Hao Guo of Iowa State University are greatly appreciated. Support from the National Science Foundation with Grant numbers of IRES-1064235 and CBET-1435590 is gratefully acknowledged.

\section{REFERENCES}

[1] D.G. Bogard, K.A. Thole, Gas Turbine Film Cooling, J. Propuls. Power. 22 (2006) 249-270. doi:10.2514/1.18034.

[2] D.G. Bogard, Airfoil film cooling, in: Gas Turbine Handb., National Energy Technology Laboratory, Section 4.2.2.1, 2006.

[3] T.F. Fric, A. Roshko, Vortical structure in the wake of a transverse jet, J. Fluid Mech. 279 (1994) 1-47. doi:10.1017/S0022112094003800.

[4] R.J. Goldstein, E.R.G. Eckert, F. Burggraf, Effects of hole geometry and density on threedimensional film cooling, Int. J. Heat Mass Transf. 17 (1974) 595-607. doi:10.1016/00179310(74)90007-6.

[5] K.M. Zaman, D. Rigby, J. Heidmann, Inclined Jet in Crossflow Interacting With a Vortex 
Generator, J. Propuls. Power. 26 (2010) 947-954.

[6] A.F. Shinn, S. Pratap Vanka, Large eddy simulations of film-cooling flows with a micro-ramp vortex generator, J. Turbomach. 135 (2013) 011004.

[7] B. An, J. Liu, C. Zhang, S. Zhou, Film Cooling of Cylindrical Hole With a Downstream Short Crescent-Shaped Block, J. Heat Transfer. 135 (2013) 031702. doi:10.1115/1.4007879.

[8] S. Na, T. Shih, Increasing adiabatic film-cooling effectiveness by using an upstream ramp, J. Heat Transfer. 129 (2007) 464-471.

[9] E. Sakai, T. Takahashi, Y. Agata, Experimental Study on Effects of Internal Ribs and Rear Bumps on Film Cooling Effectiveness, J. Turbomach. 135 (2013) 031025. doi:10.1115/1.4007546.

[10] R. Goldstein, Film cooling, in: Adv. Heat Transf. 7.1, 1971: pp. 321-379.

[11] R.S. Bunker, A Review of Shaped Hole Turbine Film-Cooling Technology, J. Heat Transfer. 127 (2005) 441. doi:10.1115/1.1860562.

[12] B. Andreotti, P. Claudin, S. Douady, Selection of dune shapes and velocities Part 1: Dynamics of sand, wind and barchans, Eur. Phys. J. B - Condens. Matter. 28 (2002) 321-339. doi:10.1140/epjb/e2002-00236-4.

[13] E. Parteli, O. Durán, H. Herrmann, Minimal size of a barchan dune, Phys. Rev. E. 75 (2007) 011301. doi:10.1103/PhysRevE.75.011301.

[14] J.A. Palmer, R. Mejia-Alvarez, J.L. Best, K.T. Christensen, Particle-image velocimetry measurements of flow over interacting barchan dunes, Exp. Fluids. 52 (2011) 809-829. doi:10.1007/s00348-011-1104-4.

[15] K.T. Christensen, F. Kazemifar, G. Blois, J.M. Barros, Z. Tang, A. Hamed, et al., Quantitative Studies of Environmental Flows at the Micro- and Macro-Scales, in: 16th Int. Symp. Flow Vis. Okinawa, Japan, 2014.

[16] B. Horvat, Barchan dunes Seminar 2, (n.d.) http://www-f1.ijs.si/ rudi/sola/Sem4.pdf.

[17] P. Hersen, K. Andersen, H. Elbelrhiti, B. Andreotti, P. Claudin, S. Douady, Corridors of barchan dunes: Stability and size selection, Phys. Rev. E. 69 (2004) 011304. doi:10.1103/PhysRevE.69.011304.

[18] S. Ou, J.-C. Han, A.B. Mehendale, C.P. Lee, Unsteady Wake Over a Linear Turbine Blade Cascade With Air and CO2 Film Injection: Part I-Effect on Heat Transfer Coefficients, J. Turbomach. 116 (1994) 721. doi:10.1115/1.2929465.

[19] J. Han, S. Dutta, S. Ekkad, Gas turbine heat transfer and cooling technology, CRC Press, Taylor \& Francis, 2012. http://books.google.com/books?hl=en\&lr=\&id=Ly66eNVjtfEC\&oi=fnd\&pg=PP1\&dq=\%5D+J.C. + +Han, + S.+Dutta,+S.+Ekkad,+Gas+Turbine+Heat+Transfer+and+Cooling+Technology,\&ots=qyF oUCqRGL\&sig=D4RS2BGXHZTQ5WhZYr_dYCchgGY (accessed August 12, 2015).

[20] S. Baldauf, M. Scheurlen, A. Schulz, S. Wittig, Correlation of Film-Cooling Effectiveness From Thermographic Measurements at Enginelike Conditions, J. Turbomach. 124 (2002) 686. doi:10.1115/1.1504443.

[21] L.M. Wright, Z. Gao, T.A. Varvel, J.-C. Han, Assessment of Steady State PSP, TSP, and IR Measurement Techniques for Flat Plate Film Cooling, in: ASME 2005 Summer Heat Transf. Conf. Collocated with ASME 2005 Pacific Rim Tech. Conf. Exhib. Integr. Packag. MEMS, NEMS, Electron. Syst. Heat Transf. Vol. 3, ASME, 2005: pp. 37-46. doi:10.1115/HT2005-72363.

[22] J.H. Bell, E.T. Schairer, L.A. Hand, R.D. Mehta, SURFACE PRESSURE MEASUREMENTS USING LUMINESCENT COATINGS 1, Annu. Rev. Fluid Mech. 33 (2001) 155-206. 
doi:10.1146/annurev.fluid.33.1.155.

[23] B. Johnson, W. Tian, K. Zhang, H. Hu, An experimental study of density ratio effects on the film cooling injection from discrete holes by using PIV and PSP techniques, Int. J. Heat Mass Transf. 76 (2014) 337-349. doi:10.1016/j.ijheatmasstransfer.2014.04.028.

[24] E.R.G. Eckert, Similarity analysis of model experiments for film cooling in gas turbines, WärmeUnd Stoffübertragung. 27 (1992) 217-223. doi:10.1007/BF01589919.

[25] J.N. Shadid, E.R.G. Eckert, The Mass Transfer Analogy to Heat Transfer in Fluids With Temperature-Dependent Properties, J. Turbomach. 113 (1991) 27. doi:10.1115/1.2927734.

[26] D. Charbonnier, P. Ott, M. Jonsson, F. Cottier, T. Köbke, Experimental and Numerical Study of the Thermal Performance of a Film Cooled Turbine Platform, Proc. Asme Turbo Expo 2009, Vol 3, Pts A B. (2009) 1027-1038.

[27] Z. Yang, H. Hu, Study of Trailing-Edge Cooling Using Pressure Sensitive Paint Technique, J. Propuls. Power. 27 (2011) 700-709. doi:10.2514/1.B34070.

[28] B. Johnson, H. Hu, Measurement Uncertainty Analysis in Determining Adiabatic Film Cooling Effectiveness by Using Pressure Sensitive Paint Technique, J. Turbomach. 138 (2016) 121004. doi:10.1115/1.4033506.

[29] M. Sajben, Uncertainty estimates for pressure sensitive paint measurements, AIAA J. 31 (1993) 2105-2110. doi:10.2514/3.11897.

[30] S.J. Kline, F.A. Mcclintock, Describing uncertainties in single sample experiments, J. Mech. Eng. 75 (1953).

[31] H. Hu, T. Saga, T. Kobayashi, N. Taniguchi, A study on a lobed jet mixing flow by using stereoscopic particle image velocimetry technique, Phys. Fluids. 13 (2001) 3425. doi:10.1063/1.1409537.

[32] D.L. Schmidt, B. Sen, D.G. Bogard, Film Cooling With Compound Angle Holes: Adiabatic Effectiveness, J. Turbomach. 118 (1996) 807. doi:10.1115/1.2840938.

[33] A.K. Sinha, D.G. Bogard, M.E. Crawford, Film-Cooling Effectiveness Downstream of a Single Row of Holes With Variable Density Ratio, J. Turbomach. 113 (1991) 442. doi:10.1115/1.2927894.

[34] D. Pedersen, Film cooling with large density differences between the mainstream and the secondary fluid measured by the heat-mass transfer analogy, J. Heat Transfer. 99 (1977) 620-627.

[35] Y. Zheng, A. Rinoshika, Multi-scale vortical structure analysis on large eddy simulation of dune wake flow, J. Vis. 18 (2015) 95-109. doi:10.1007/s12650-014-0227-0.

[36] M. Omidyeganeh, U. Piomelli, K.T. Christensen, J.L. Best, Large-eddy simulation of flow over barchan dunes, in: Mar. River Dune Dyn. - MARID IV, Bruges, 2013: pp. 191-198.

[37] D.J. Needham, N. Riley, J.H.B. Smith, A jet in crossflow, J. Fluid Mech. 188 (1988) 159. doi:10.1017/S0022112088000680.

[38] M. Ilak, P. Schlatter, S. Bagheri, D.S. Henningson, Bifurcation and stability analysis of a jet in cross-flow: onset of global instability at a low velocity ratio, J. Fluid Mech. 696 (2012) 94-121. doi:10.1017/jfm.2012.10.

[39] D.R. Getsinger, L. Gevorkyan, O.I. Smith, A.R. Karagozian, Structural and stability characteristics of jets in crossflow, J. Fluid Mech. 760 (2014) 342-367. doi:10.1017/jfm.2014.605. 